ESAIM: M2AN

Vol. 40, No 5, 2006, pp. 939-960

DOI: $10.1051 / \mathrm{m} 2 \mathrm{an}: 2006037$
ESAIM: Mathematical Modelling and Numerical Analysis

www.edpsciences.org $/ \mathrm{m} 2$ an

\title{
CONSERVATION LAW CONSTRAINED OPTIMIZATION BASED UPON FRONT-TRACKING
}

\author{
Martin Gugat ${ }^{1}$, Michä̈l Herty ${ }^{2}$, Axel Klar ${ }^{2}$ AND \\ GUNTER LEUGERING ${ }^{1}$
}

\begin{abstract}
We consider models based on conservation laws. For the optimization of such systems, a sensitivity analysis is essential to determine how changes in the decision variables influence the objective function. Here we study the sensitivity with respect to the initial data of objective functions that depend upon the solution of Riemann problems with piecewise linear flux functions. We present representations for the onesided directional derivatives of the objective functions. The results can be used in the numerical method called Front-Tracking.
\end{abstract}

Mathematics Subject Classification. 35Lxx, 76N15.

Received: November 15, 2005.

\section{INTRODUCTION}

Conservation laws play an important role in physics and engineering. They appear for example as a model of traffic flow $[1,8,12,17]$, in two phase flow problems [16], in water flows $[6,7]$ and gas dynamics $[4,16,18]$. Usually, certain system parameters can be controlled and therefore, the necessity arises to deal with problems of optimal control. In general the optimal control problem is driven by nonlinear conservation laws and to obtain gradient information we need a sensitivity calculus that incorporates those nonlinear hyperbolic equations.

We consider a sensitivity calculus for scalar nonlinear hyperbolic conservation laws with piecewise linear flux and piecewise constant initial data. This setting was introduced by Dafermos in [3]. Later different authors extended the introduced ideas and developed a scheme, called front- [13] or wave-tracking [2] which is at one hand an analytical tool for proving existence and uniqueness of solutions, and at the other hand an efficient numerical method. For further references, see $[2,11,13,14]$.

For effective numerical computations we derive a sensitivity calculus for nonlinear hyperbolic equations in the front-tracking setting. The numerical method computes the state and a consistent gradient. In terms of the Front-Tracking method, the additional effort for the gradient computation is neglectable. In a forthcoming publication we will present numerical examples using the theoretical results developed here. Also the theoretical results are valid for non-convex and non-concave flux functions.

Related work on sensitivity analysis for networked hyperbolic systems within the framework of continuously differentiable solutions has been given in [5]. A sensitivity analysis for nonlinear

\footnotetext{
Keywords and phrases. Sensitivity calculus, front-tracking, conservation laws.

1 Institut für Angewandte Mathematik, FAU Erlangen-Nürnberg, Martensstr. 3, 91058 Erlangen, Germany.

2 Fachbereich Mathematik, TU Kaiserslautern, Postfach 3049, 67653 Kaiserslautern, Germany.
} 
hyperbolic conservation laws with source term has been given by Ulbrich in $[19,20]$. We perform this analysis in the framework of front-tracking, since this yields explicit representations of the derivatives that can be used numerically.

In Section 2 we recall the existence result for a scalar conservation law with piecewise linear flux function due to Dafermos [3], since the subsequent analysis is based on this result. In Section 3 we introduce the optimization problem under consideration and state the main result of the discussion, namely, the cost functional allows for a first-order expansion. In Section 4 we show how the main result of the previous section can be incorporated in the Front-Tracking algorithm for numerically solving the optimization problem, see also the example in Section 9. The proof of the main result is split among Sections 5 to 8. Therein, Section 5 is devoted to estimate the change in breakpoints of the flux function for sufficiently small changes in the initial value. Section 6 contains the first-order expansion of piecewise linear functions. This results are used to proof the first-order expansion in the state variable in Section 7. Finally, in Section 8 we combine the previous results and proof the main result. In Section 9 we apply the derived result to two simple examples related to traffic flow and Burger's equation, before we conclude our presentation with a summary and an outlook in Section 10.

\section{Cauchy problem}

We consider Cauchy problems on the real line. Due to Dafermos [3] we have

Lemma 2.1. Assume $f$ is piecewise linear, Lipschitz continuous on $[m, M]$ and

$$
u_{0}(x)= \begin{cases}u_{l} & x \leq 0 \\ u_{r} & x>0\end{cases}
$$

where $u_{l}, u_{r} \in[m, M]$ are constants. Then there exists an entropic weak solution of

$$
y_{t}(x, t)+f(y(x, t))_{x}=0
$$

and

$$
y(x, 0)=u_{0}(x)
$$

which consists of a finite number of constants states separated by shocks centered at the origin.

We consider entropy weak solutions in the sense of Kruzkov, see [15]. An introduction to this type of solutions is given in [13].

The solution can be given explicitly by

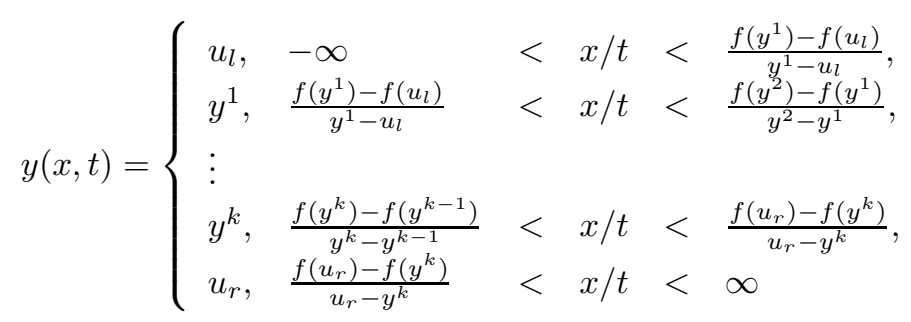

where the points $y^{k}$ are determined as follows. In the case $u_{l}<u_{r}$ the boundary of the convex hull of the set

$$
\left\{(y, v): u_{l} \leq y \leq u_{r}, v \geq f(y)\right\}
$$

is a polygonal line with vertices at $\left(u_{l}, f\left(u_{l}\right)\right),\left(y^{1}, f\left(y^{1}\right)\right), \ldots,\left(y^{k}, f\left(y^{k}\right)\right),\left(u_{r}, f\left(u_{r}\right)\right)$ for $u_{l}<y^{1}<$ $\cdots<y^{k}<u_{r}$. Herein $\left(y^{i}, f\left(y^{i}\right)\right), i=1, \ldots, k$ are also vertices of the graph of $f$. The construction is similar for the case $u_{r}<u_{l}$. 
Let $h=\min \left\{\left|y^{i+1}-y^{i}\right|: i=1, \ldots, k-1\right\}, \delta=\left(\delta_{1}, \delta_{2}\right)$ with $\|\delta\|_{\infty}<h$. We consider disturbed initial data

$$
u_{0}^{\delta}(x)= \begin{cases}u_{l}+\delta_{1} & x \leq 0 \\ u_{r}+\delta_{2} & x>0\end{cases}
$$

and the weak admissible solution $y^{\delta}$ to (2), see Section 4 for a description of the Front-Tracking algorithm based on the previous results.

\section{Main Result: SENSitivity CALCUlus FOR THE OBJECTIVE FUnCTION}

We introduce the general optimal control problems for our discussion. We assume the objective functions of the following form, i.e., terminal measurement on a (sub-)set of the real line. Let $x_{1}<0<x_{2}$ and $T>0$ be given. Let $g$ be a continuously differentiable real-valued function on $\mathbb{R}$ with compact support. Define

$$
I\left(u_{0}\right)=\int_{x_{1}}^{x_{2}} g(y(x, T)) \mathrm{d} x
$$

where $y$ is the solution of the Cauchy problem (2),(3). We derive a sensitivity calculus for $I$ in Theorem 3.2 below. The corresponding optimization problem is

$$
\min _{u_{0}} I\left(u_{0}\right) .
$$

For the solution of (8), a sensitivity calculus for the objective function $I$ is essential. In general only the one-sided directional derivatives of $I$ exist.

Definition 3.1. For $\delta \in \mathbb{R}^{2}$ with

$$
m<u_{l}+\delta_{1}<u_{r}+\delta_{2}<M
$$

let $f_{u}^{\delta}$ denote the lower convex envelope of $f$ in the interval $\left[u_{l}+\delta_{1}, u_{r}+\delta_{2}\right]$. In particular, $f_{u}^{0}$ is the lower convex envelope of $f$ in the interval $\left[u_{l}, u_{r}\right]$.

Theorem 3.2. Let I be as in (7). Let $u_{0}$ be as in (1) and $y_{0}(x, t)$ be the solution to the Cauchy problem (2),(3). Let $u_{0}^{\delta}$ be as in (6).

Assume that $u_{l}, u_{r} \in(m, M), u_{l}<u_{r}$. Assume that $k \geq 1$.

For $\delta \in \mathbb{R}^{2}$, define

$$
h(\delta)=I\left(u_{0}^{\delta}\right) .
$$

Then the following expansion holds:

$$
I\left(u_{0}^{\delta}\right)=I\left(u_{0}\right)+\delta_{1} \partial_{\delta_{1} \pm} h(0)+\delta_{2} \partial_{\delta_{2} \pm} h(0)+O\left(\|\delta\|^{2}\right)
$$

where the right-hand side derivatives $\partial_{\delta_{1}+}, \partial_{\delta_{2}+}$ or the left-hand side derivatives $\partial_{\delta_{1}-}, \partial_{\delta_{2}-}$ are chosen according to the sign of $\delta_{1}, \delta_{2}$ respectively. The one-sided partial derivatives of $h$ are given below.

Let

$$
D_{l}=\frac{f\left(y_{1}\right)-f\left(u_{l}\right)}{y_{1}-u_{l}}, D_{r}=\frac{f\left(u_{r}\right)-f\left(y_{k}\right)}{u_{r}-y_{k}} .
$$

Let $\chi_{\left[x_{1}, x_{2}\right)}$ denote the characteristic function of the interval $\left[x_{1}, x_{2}\right)$ that is

$$
\chi_{\left[x_{1}, x_{2}\right)}(x)=\left\{\begin{array}{lll}
0 & \text { if } & x \notin\left[x_{1}, x_{2}\right) \\
1 & \text { if } & x \in\left[x_{1}, x_{2}\right)
\end{array}\right.
$$

For an interval $[a, b]$, let $\mu([a, b])=b-a$ denote the length of the interval. 
Computation of $\partial_{\delta_{1}} h(0)$.

If $D_{l}-f_{-}^{\prime}\left(u_{l}\right) \leq 0$ holds, then

$$
\partial_{\delta_{1}-} h(0)=g^{\prime}\left(u_{l}\right) \mu\left(\left[x_{1}, x_{2}\right] \cap\left(-\infty, T D_{l}\right]\right)-\frac{g\left(y_{1}\right)-g\left(u_{l}\right)}{y_{1}-u_{l}} T\left[D_{l}-f_{-}^{\prime}\left(u_{l}\right)\right] \chi_{\left[x_{1}, x_{2}\right)}\left(T D_{l}\right) .
$$

If $D_{l}-f_{-}^{\prime}\left(u_{l}\right)>0$ then

$$
\partial_{\delta_{1}-} h(0)=g^{\prime}\left(u_{l}\right) \mu\left(\left[x_{1}, x_{2}\right] \cap\left(-\infty, T f_{-}^{\prime}\left(u_{l}\right)\right]\right) .
$$

Computation of $\partial_{\delta_{1}+} h(0)$.

If $\left\{D_{l}=f_{+}^{\prime}\left(u_{l}\right)\right.$ or for all $\left.u \in\left(u_{l}, y_{1}\right): f(u)>f_{u}^{0}(u)\right\}$ holds, then

$$
\partial_{\delta_{1}+} h(0)=g^{\prime}\left(u_{l}\right) \mu\left(\left[x_{1}, x_{2}\right] \cap\left(-\infty, T D_{l}\right]\right)-\frac{g\left(y_{1}\right)-g\left(u_{l}\right)}{y_{1}-u_{l}} T\left[D_{l}-f_{+}^{\prime}\left(u_{l}\right)\right] \chi_{\left(x_{1}, x_{2}\right]}\left(T D_{l}\right) .
$$

If $\left\{D_{l} \neq f_{+}^{\prime}\left(u_{l}\right)\right.$ and there exists $\left.u \in\left(u_{l}, y_{1}\right): f(u)=f_{u}^{0}(u)\right\}$,

let $\bar{u}=\inf \left\{u \in\left(u_{l}, y_{1}\right): f(u)=f_{u}^{0}(u)\right\}$. Then

$$
\partial_{\delta_{1}+} h(0)=g^{\prime}\left(u_{l}\right) \mu\left(\left[x_{1}, x_{2}\right] \cap\left(-\infty, T D_{l}\right]\right)-\frac{g(\bar{u})-g\left(u_{l}\right)}{\bar{u}-u_{l}} T\left[D_{l}-f_{+}^{\prime}\left(u_{l}\right)\right] \chi_{\left(x_{1}, x_{2}\right]}\left(T D_{l}\right) .
$$

Computation of $\partial_{\delta_{2}-} h(0)$.

If $\left\{D_{r}=f_{-}^{\prime}\left(u_{r}\right)\right.$ or for all $\left.u \in\left(y_{k}, u_{r}\right): f(u)>f_{u}^{0}(u)\right\}$ holds, then

$$
\partial_{\delta_{2}-} h(0)=g^{\prime}\left(u_{r}\right) \mu\left(\left[x_{1}, x_{2}\right] \cap\left[T D_{r}, \infty\right)\right)+\frac{g\left(y_{k}\right)-g\left(u_{r}\right)}{y_{k}-u_{r}} T\left[D_{r}-f_{-}^{\prime}\left(u_{r}\right)\right] \chi_{\left[x_{1}, x_{2}\right)}\left(T D_{r}\right) .
$$

If $\left\{D_{r} \neq f_{-}^{\prime}\left(u_{r}\right)\right.$ and there exists $\left.u \in\left(y_{k}, u_{r}\right): f(u)=f_{u}^{0}(u)\right\}$,

let $\bar{u}=\sup \left\{u \in\left(y_{k}, u_{r}\right): f(u)=f_{u}^{0}(u)\right\}$. Then

$$
\partial_{\delta_{2}-} h(0)=g^{\prime}\left(u_{r}\right) \mu\left(\left[x_{1}, x_{2}\right] \cap\left[T D_{r}, \infty\right)\right)+\frac{g(\bar{u})-g\left(u_{r}\right)}{\bar{u}-u_{r}} T\left[D_{r}-f_{-}^{\prime}\left(u_{r}\right)\right] \chi_{\left[x_{1}, x_{2}\right)}\left(T D_{r}\right) .
$$

Computation of $\partial_{\delta_{2}} h(0)$.

If $f_{+}^{\prime}\left(u_{r}\right) \leq D_{r}$ holds, then

$$
\partial_{\delta_{2}+} h(0)=g^{\prime}\left(u_{r}\right) \mu\left(\left[x_{1}, x_{2}\right] \cap\left[T D_{r}, \infty\right)\right)+\frac{g\left(u_{r}\right)-g\left(y_{k}\right)}{u_{r}-y_{k}} T\left[D_{r}-f_{+}^{\prime}\left(u_{r}\right)\right] \chi_{\left(x_{1}, x_{2}\right]}\left(T D_{r}\right) .
$$

If $f_{+}^{\prime}\left(u_{r}\right)>D_{r}$ then

$$
\partial_{\delta_{2}+} h(0)=g^{\prime}\left(u_{r}\right) \mu\left(\left[x_{1}, x_{2}\right] \cap\left[T f_{+}^{\prime}\left(u_{r}\right), \infty\right)\right) .
$$

Remark 3.3. Theorem 3.2 holds true even for a non-convex objective function. In the case $k=0$ the assertion of the theorem is true, once we replace $y_{k}=u_{l}$ and $y_{1}=u_{r}$ at the appropriate places.

Although our objective function is of integral type, in the derivative integrals do not appear explicitly. However, the terms where measures $\mu$ appear can obviously be interpreted as integrals. This is not the case for the additional shift-terms where the characteristic functions and the shock speeds appear. 


\section{Front-Tracking for the Cauchy problem}

The main result is based on the Front-Tracking algorithm for solving conservation laws. A detailed description of this method with analysis of convergence rates and discussion of properties can be found e.g. in $[2,3,13]$ and in particular in publications of the Norwegian school $[10,11,14]$. For convenience we recall the Front-Tracking method as presented in Chapter 2 of [13] (see also the internet page of K.-A. Lie: http://www.math.ntnu.no/ andreas/fronttrack/ftrack/implement.html): Given a scalar onedimensional conservation law as in (2) and initial data $u_{0}(x)$ piecewise constant and the flux function $y \rightarrow f(y)$ being continuous, piecewise linear (as in the assertion of Lem. 2.1). Assume that $f(y)$ has breakpoints $\left\{y_{i}\right\}, i=1,2, \ldots$ and that $u_{0}$ takes values in the set $\left\{y_{i}\right\}$. Then, the Front-Tracking algorithm can be stated as follows:

\section{WHILE $T<\infty$}

(1) Solve Riemann problems and compute possible collisions between fronts;

(2) $\mathrm{T}$ = first.collision.time;

(3) remove colliding fronts;

(4) solve corresponding Riemann problem;

(5) insert new fronts;

(6) compute possible new collisions;

(7) $\mathrm{T}=$ next.collision.time;

\section{END}

Some remarks on the scheme are in order. First, the scheme is grid-free and only the traveling fronts have to be tracked throughout the simulation. Second, the scheme is first-order only. Third, at each time $t$ the solution $y(x, t)$ consists of finite number of traveling discontinuities. Last, comparing with the result on sensitivity, we see that the sensitivities for cost functional as given by (7) can be computed from the Front-Tracking approximation: indeed, consider the equations (11)-(16) for $\partial_{\delta_{1,2}^{ \pm}} h(0)$. Therein, only the objective function, the approximate derivative $D_{l, r} f$ and the one-sided derivatives $f_{ \pm}^{\prime}$ appear. All the values are known at each time $t>0$ for the piecewise constant solution $y(x, t)$ of the Front-Tracking procedure. Hence, there are no additional equations to solve for computing sensitivities beyond the standard Front-Tracking code. In particular, this sensitivity calculus is then also grid-free.

\section{Convex envelope of Piecewise linear FunCtions}

For our analysis, we need a sensitivity Lemma about the behavior of the lower convex envelope of piecewise linear functions if the interval where the convex envelope is changed. It turns out that if the changes of the boundary points of the interval are small enough, the resulting changes in the convex hull are easy to describe: first, changes on the left-hand side are independent of changes on the right-hand side. Moreover, on each side either the number of breakpoints remains constant (in this case the first breakpoint is moved), or one additional breakpoint appears. As a breakpoint of a function $f$ we understand a vertex of the lower convex envelope of $f$. To be more precise, we define:

Definition 5.1. Let $f: \mathbb{R} \rightarrow[m, M]$ be a given piecewise linear function.

$u \in \mathbb{R}$ is called a breakpoint of $f$, if $(u, f(u))$ is a vertex of the lower convex envelope of $f$.

Lemma 5.2. Let real numbers $m, M$ be given such that $m<M$. Let $u_{l}, u_{r} \in(m, M)$ be given such that $u_{l}<u_{r}$. Consider a piecewise linear function $f$ that is defined on $[m, M]$.

Then $f_{u}^{\delta}$ is piecewise linear and the set of breakpoints of $f_{u}^{\delta}$ is contained in the set of breakpoints of $f$. Assume that $f_{u}^{0}$ has at least one breakpoint in $\left(u_{l}, u_{r}\right)$. Let $y_{1}$ denote the smallest breakpoint of $f_{u}^{0}$ that is larger than $u_{l}$. Let $y_{k}$ denote the largest breakpoint of $f_{u}^{0}$ that is smaller than $u_{r}$.

If $\left|\delta_{1}\right|+\left|\delta_{2}\right|$ is sufficiently small, then between $y_{1}$ and $y_{k}$ the function $f_{u}^{\delta}$ has the same breakpoints as $f_{u}^{0}$. 

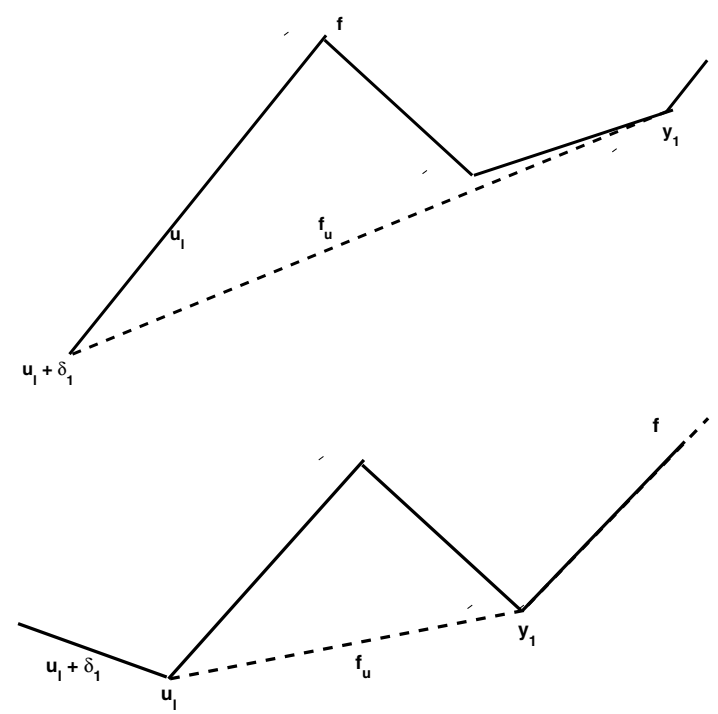

Figure 1. Example for the case $\delta_{1}<0$. Top part corresponds to the case where condition (19) is satisfied. Bottom part shows a case where condition (19) is violated and where we obtain an additional breakpoint.

\section{Case: The first breakpoint is moved. If}

$$
\delta_{1}<0 \text { and } f_{-}^{\prime}\left(u_{l}\right) \geq\left(f_{u}^{0}\right)_{+}^{\prime}\left(u_{l}\right),
$$

then $u_{l}+\delta_{1}$ is the only breakpoint of $f_{u}^{\delta}$ that is smaller than $y_{1}$. This is also the case if $\delta_{1}>0$ and

$$
\left\{f_{+}^{\prime}\left(u_{l}\right)=\left(f_{u}^{0}\right)_{+}^{\prime}\left(u_{l}\right) \text { or for all } u \in\left(u_{l}, y_{1}\right) \text { we have } f(u)>f_{u}^{0}(u)\right\} \text {. }
$$

Case: Additional breakpoint in $\left(-\infty, y_{1}\right]$. Otherwise $f_{u}^{\delta}$ has two breakpoints $u_{l}+\delta_{1}, \bar{u}$ that are smaller than $y_{1}$. If $\delta_{1}<0, \bar{u}=u_{l}$. If $\delta_{1}>0$, the point $\bar{u}$ is the smallest breakpoint of $f$ in $\left(u_{l}, y_{1}\right)$ such that on $\left[\bar{u}, y_{1}\right]$ we have $f_{u}^{0}=f$.

Case: The last breakpoint is moved. If

$$
\delta_{2}>0 \text { and } f_{+}^{\prime}\left(u_{r}\right) \leq\left(f_{u}^{0}\right)_{-}^{\prime}\left(u_{r}\right),
$$

then $u_{r}+\delta_{2}$ is the only breakpoint of $f_{u}^{\delta}$ that is larger than $y_{k}$. This is also the case if $\delta_{2}<0$ and

$$
\left\{f_{-}^{\prime}\left(u_{r}\right)=\left(f_{u}^{0}\right)_{-}^{\prime}\left(u_{r}\right) \text { or for all } u \in\left(y_{k}, u_{r}\right) \text { we have } f(u)>f_{u}^{0}(u)\right\} \text {. }
$$

Case: Additional breakpoint in $\left[y_{k}, \infty\right)$. Otherwise $f_{u}^{\delta}$ has two breakpoints $\bar{u}, u_{r}+\delta_{2}$ that are larger than $y_{k}$. If $\delta_{2}>0, \bar{u}=u_{r}$. If $\delta_{2}<0$, the point $\bar{u}$ is the largest breakpoint of $f$ in $\left(y_{k}, u_{r}\right)$ such that on $\left[y_{k}, u_{r}\right]$ we have $f_{u}^{0}=f$.

Before we start with the proof of the Lemma we provide two pictures, that should motivate the discussion below. The notation in Figures 1 and 2 are as in the above lemma.

Proof. The epigraph of $f_{u}^{\delta}$ is the convex hull of the epigraph of $f$. Since $f$ is piecewise linear, the convex hull of the epigraph of $f$ has a finite number of vertices. These vertices are breakpoints of $f$. This implies that $f_{u}^{\delta}$ is also piecewise linear and the set of breakpoints of $f_{u}^{\delta}$ is contained in the set of breakpoints of $f$. 


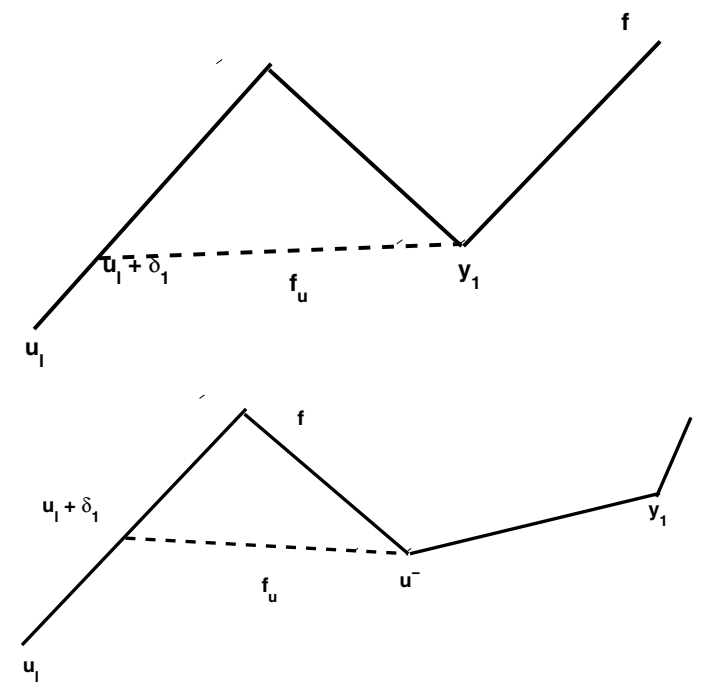

Figure 2. Example for the case $\delta_{1}>0$. Top part of the figure shows a case where (20) holds and the bottom part gives an example for a case where we obtain an additional breakpoint $\bar{u}$ of $f$.

Choose $\left|\delta_{1}\right|>0$ so small that $f$ is linear between $u_{l}$ and $u_{l}+\delta_{1}$. Choose $\left|\delta_{2}\right|>0$ so small that $f$ is linear between $u_{r}$ and $u_{r}+\delta_{2}$.

We have

$$
\frac{f\left(y_{1}\right)-f\left(u_{l}\right)}{y_{1}-u_{l}}=\left(f_{u}^{0}\right)_{+}^{\prime}\left(u_{l}\right)=\left(f_{u}^{0}\right)_{-}^{\prime}\left(y_{1}\right)<\left(f_{u}^{0}\right)_{+}^{\prime}\left(y_{1}\right) .
$$

Thus we can choose $\left|\delta_{1}\right|$ so small that we have

$$
\frac{f\left(y_{1}\right)-f\left(u_{l}+\delta_{1}\right)}{y_{1}-u_{l}-\delta_{1}}<\left(f_{u}^{0}\right)_{+}^{\prime}\left(y_{1}\right) .
$$

We have

$$
\frac{f\left(u_{r}\right)-f\left(y_{k}\right)}{u_{r}-y_{k}}=\left(f_{u}^{0}\right)_{-}^{\prime}\left(u_{r}\right)=\left(f_{u}^{0}\right)_{+}^{\prime}\left(y_{k}\right)>\left(f_{u}^{0}\right)_{-}^{\prime}\left(y_{k}\right) .
$$

Thus we can choose $\left|\delta_{2}\right|$ so small that we have

$$
\frac{f\left(u_{r}+\delta_{2}\right)-f\left(y_{k}\right)}{u_{r}+\delta_{2}-y_{k}}>\left(f_{u}^{0}\right)_{-}^{\prime}\left(y_{k}\right) .
$$

Since $\left(f_{u}^{0}\right)_{+}^{\prime}\left(u_{l}\right)<\left(f_{u}^{0}\right)_{-}^{\prime}\left(u_{r}\right)$, we can choose $\left|\delta_{1}\right|+\left|\delta_{2}\right|$ so small that

$$
\frac{f\left(y_{1}\right)-f\left(u_{l}+\delta_{1}\right)}{y_{1}-u_{l}-\delta_{1}}<\frac{f\left(u_{r}+\delta_{2}\right)-f\left(y_{k}\right)}{u_{r}+\delta_{2}-y_{k}} .
$$

5.1. The case $\delta_{1}<0, \delta_{2}>0$

Now we consider the case $\delta_{1}<0, \delta_{2}>0$ where $\left[u_{l}, u_{r}\right] \subset\left[u_{l}+\delta_{1}, u_{r}+\delta_{2}\right]$.

Consider the polygonal line through the points

$$
\left(u_{l}+\delta_{1}, f\left(u_{l}+\delta_{1}\right)\right),\left(u_{l}, f\left(u_{l}\right)\right),\left(y_{1}, f\left(y_{1}\right)\right) \text {. }
$$

If it is the graph of a convex function, then the polygonal line through

$$
\left(u_{l}+\delta_{1}, f\left(u_{l}+\delta_{1}\right)\right),\left(u_{l}, f\left(u_{l}\right)\right),\left(y_{1}, f\left(y_{1}\right)\right), \ldots,\left(y_{k}, f\left(y_{k}\right)\right)
$$


is also the graph of a convex function $g_{1}$. Moreover, $g_{1} \leq f$ on $\left[u_{l}+\delta_{1}, y_{k}\right]$.

If the polygonal line through the points (24) is not the graph of a convex function (this is the case if (19) holds), then the polygonal line through the points

$$
\left(u_{l}+\delta_{1}, f\left(u_{l}+\delta_{1}\right)\right),\left(y_{1}, f\left(y_{1}\right)\right), \ldots,\left(y_{k}, f\left(y_{k}\right)\right)
$$

is the graph of a convex function $g_{1}$. Moreover, $g_{1} \leq f$ on $\left[u_{l}+\delta_{1}, y_{k}\right]$.

Now consider the polygonal line through

$$
\left(y_{k}, f\left(y_{k}\right)\right),\left(u_{r}, f\left(u_{r}\right)\right),\left(u_{r}+\delta_{2}, f\left(u_{r}+\delta_{2}\right)\right) .
$$

If it is the graph of a convex function, then also the polygonal line through

$$
\left(y_{1}, f\left(y_{1}\right)\right), \ldots,\left(y_{k}, f\left(y_{k}\right)\right),\left(u_{r}, f\left(u_{r}\right)\right),\left(u_{r}+\delta_{2}, f\left(u_{r}+\delta_{2}\right)\right)
$$

is the graph of a convex function $g_{2}$. Moreover, $g_{2} \leq f$ on $\left[y_{1}, u_{r}+\delta_{2}\right]$.

If the polygonal line through the points (25) is not the graph of a convex function (this is the case if (21) holds), then the polygonal line through the points

$$
\left(y_{1}, f\left(y_{1}\right)\right), \ldots,\left(y_{k}, f\left(y_{k}\right)\right),\left(u_{r}+\delta_{2}, f\left(u_{r}+\delta_{2}\right)\right)
$$

is the graph of a convex function $g_{2}$ with $g_{2} \leq f$ on $\left[y_{1}, u_{r}+\delta_{2}\right]$.

Thus we have constructed convex functions $g_{1}, g_{2}$ with $g_{1}=g_{2}$ on $\left[y_{1}, y_{k}\right]$.

Define the function $g$ on $\left[u_{l}+\delta_{1}, u_{r}+\delta_{2}\right]$ by $g(u)=g_{1}(u)$ for $u \in\left[u_{l}+\delta_{1}, y_{1}\right]$ and $g(u)=g_{2}(u)$ for $u>y_{1}$.

Then $g$ is convex on $\left[u_{l}+\delta_{1}, u_{r}+\delta_{2}\right]$. (If $y_{1}=y_{k}$, this is due to (23).) Moreover, $g \leq f$ on $\left[u_{l}+\delta_{1}, u_{r}+\delta_{2}\right]$. By our construction, at the breakpoints of $g$ we have $f(u)=g(u)$. This implies that $g=f_{u}^{\delta}$.

\subsection{The case $\delta_{1}>0, \delta_{2}>0$}

Now we consider the case $\delta_{1}>0, \delta_{2}>0$.

Assume that $(20)$ holds. If $f_{+}^{\prime}\left(u_{l}\right)=\left(f_{u}^{0}\right)_{+}^{\prime}\left(u_{l}\right)$, we define the convex function $g_{3}(u)=f_{u}^{0}$ on $\left[u+\delta_{1}, \ldots, y_{k}\right]$. Then $g_{3}(u) \leq f$ on $\left[u+\delta_{1}, \ldots, y_{k}\right]$ and $g_{3}\left(u+\delta_{1}\right)=f\left(u+\delta_{1}\right)$.

If $f_{+}^{\prime}\left(u_{l}\right)>\left(f_{u}^{0}\right)_{+}^{\prime}\left(u_{l}\right)$, condition (20) implies that for all $u \in\left(u_{l}, y_{1}\right)$, we have $f(u)>f_{u}^{0}(u)$. Hence if $\delta_{1}$ is sufficiently small, the line through $\left(u_{l}+\delta_{1}, f\left(u_{l}+\delta_{1}\right)\right),\left(y_{1}, f\left(y_{1}\right)\right)$ is below the graph of $f$. Thus for the convex function $g_{3}$ whose graph is the polygonal line through $\left(u_{l}+\delta_{1}, f\left(u_{l}+\delta_{1}\right)\right)$, $\left(y_{1}, f\left(y_{1}\right)\right), \ldots,\left(y_{k}, f\left(y_{k}\right)\right)$ we have $g_{3}(u) \leq f$ on $\left[u+\delta_{1}, \ldots, y_{k}\right]$.

If (20) is violated, then

$$
f_{+}^{\prime}\left(u_{l}\right)>\left(f_{u}^{0}\right)_{+}^{\prime}\left(u_{l}\right)
$$

and there exist breakpoints of $f$ in $\left(u_{l}, y_{1}\right)$ such that on $\left[\bar{u}, y_{1}\right]$ we have $f_{u}^{0}=f$. Let $\bar{u}$ be the smallest breakpoint of this type. Consider the polygonal line through the points

$$
\left.\left(u_{l}+\delta_{1}\right), f\left(u_{l}+\delta_{1}\right)\right),(\bar{u}, f(\bar{u})),\left(y_{1}, f\left(y_{1}\right)\right) .
$$

Since $f$ is linear on the interval $\left[u_{l}, u_{l}+\delta_{1}\right]$, condition $(26)$ implies that we have

$$
\frac{f(\bar{u})-f\left(u_{l}+\delta_{1}\right)}{\bar{u}-u_{1}-\delta_{1}}<\frac{f(\bar{u})-f\left(u_{l}\right)}{\bar{u}-u_{l}}=\left(f_{u}^{0}\right)_{+}^{\prime}\left(u_{l}\right) .
$$

Since

$$
\left(f_{u}^{0}\right)_{+}^{\prime}\left(u_{l}\right)=\frac{f\left(y_{1}\right)-f(\bar{u})}{y_{1}-\bar{u}}
$$

(28) shows that the polygonal line through the points (27) is the graph of a convex function $h$. Moreover, we can choose $\left|\delta_{1}\right|$ so small that on the interval $\left[u_{l}+\delta_{1}, y_{1}\right]$ we have $h \leq f$. Thus we 
can define the convex function $g_{3}$ on $\left[u_{l}+\delta_{1}, y_{k}\right]$ as the function whose graph is the polygonal line through the points

$$
\left.\left(u_{l}+\delta_{1}\right), f\left(u_{l}+\delta_{1}\right)\right),(\bar{u}, f(\bar{u})),\left(y_{1}, f\left(y_{1}\right)\right), \ldots,\left(y_{k}, f\left(y_{k}\right)\right)
$$

Then we have $g_{3}(u) \leq f$ on $\left[u+\delta_{1}, \ldots, y_{k}\right]$.

Now define the function $g_{2}$ as in Section 5.1. Then we define the function $g$ on $\left[u_{l}+\delta_{1}, u_{r}+\delta_{2}\right]$ by $g(u)=g_{3}(u)$ for $u \in\left[u_{l}+\delta_{1}, y_{1}\right]$ and $g(u)=g_{2}(u)$ for $u>y_{1}$. As in Section 5.1, we see that $g$ is a convex function and $g=f_{u}^{\delta}$.

\subsection{Other cases}

The other two cases $\left(\delta_{1}>0, \delta_{2}<0\right)$ and $\left(\delta_{1}<0, \delta_{2}<0\right)$ are treated analogously by constructing convex function on $\left[u_{l}+\delta_{1}, y_{k}\right]$ and $\left[y_{1}, u_{r}+\delta_{2}\right]$ that are the lower convex envelope of $f$ on these intervals. In fact, these two functions are equal to the lower convex envelope of $f$ on $\left[u_{l}+\delta_{1}, u_{r}+\delta_{2}\right]$ on their respective domains.

\section{SECOND ORDER EXPANSION OF PIECEWISE LINEAR FUNCTIONS}

We also need the following Lemma about piecewise differentiable functions, is a statement about their first derivative.

Lemma 6.1. Let $f$ be a piecewise linear function. Let $u, y \in \mathbb{R}, u \neq y$ be given. Define

$$
D=\frac{f(y)-f(u)}{y-u}
$$

Assume that $|\delta|$ is such that $f$ is linear between $u+\delta$ and $u$.

If $\delta<0$, we have

$$
\frac{f(y)-f(u+\delta)}{y-u-\delta}=D+\delta \frac{D-f_{-}^{\prime}(u)}{y-u}+\frac{\delta^{2}}{(y-u)(y-u-\delta)}\left[D-f_{-}^{\prime}(u)\right] .
$$

If $\delta>0$, we have

$$
\frac{f(y)-f(u+\delta)}{y-u-\delta}=D+\delta \frac{D-f_{+}^{\prime}(u)}{y-u}+\frac{\delta^{2}}{(y-u)(y-u-\delta)}\left[D-f_{+}^{\prime}(u)\right] .
$$

Proof. Let $\delta<0$.

$$
\begin{aligned}
\frac{f(y)-f(u+\delta)}{y-u-\delta}-D & =\frac{f(y)-f(u+\delta)}{y-u-\delta}-\frac{f(y)-f(u)}{y-u} \\
& =\frac{[f(y)-f(u+\delta)][y-u]-[f(y)-f(u)][y-u-\delta]}{[y-u-\delta][y-u]} \\
& =\frac{[f(y)-f(u)] \delta+[f(u)-f(u+\delta)][y-u]}{[y-u-\delta][y-u]} \\
& =\delta \frac{D}{y-u-\delta}-\delta \frac{f(u)-f(u+\delta)}{-\delta} \frac{1}{y-u-\delta} \\
& =\delta\left[D-f_{-}^{\prime}(u)\right] \frac{1}{y-u-\delta} \\
& =\delta \frac{D-f_{-}^{\prime}(u)}{y-u}\left[1+\frac{\delta}{y-u-\delta}\right] .
\end{aligned}
$$

The proof for $\delta>0$ works analogously. 


\section{Approximation of the DeRivative of the State}

Here we derive an approximation of the derivative of $y$ with respect to $u_{0}$.

We want to prove that a first-order expansion of $u^{\delta}$ with respect to $\delta$ exists.

Theorem 7.1. Assume that $u_{l}, u_{r} \in(m, M), u_{l}<u_{r}$. Assume that $k \geq 1$. There exists a function $H^{\delta}(x, t)$ of $\delta, x, t$ and such that for $\|\delta\|$ sufficiently small

$$
\left\|y^{\delta}-\left(y+H^{\delta}\right)\right\|_{L^{1}((0, T) \times \mathbb{R})}=O\left(\|\delta\|^{2}\right)
$$

and $H^{\delta}$ can be computed in the following way:

$$
\begin{aligned}
& H^{\delta}=H_{l-}^{\delta}+H_{r+}^{\delta} \text { if } \delta_{1} \leq 0 \text { and } \delta_{2} \geq 0, \\
& H^{\delta}=H_{l+}^{\delta}+H_{r+}^{\delta} \text { if } \delta_{1} \geq 0 \text { and } \delta_{2} \geq 0,
\end{aligned}
$$

etc., with $H_{l-}^{\delta}, H_{l+}^{\delta}, H_{r-}^{\delta}, H_{r+}^{\delta}$ defined in the following way:

we have

$$
\text { If } f_{-}^{\prime}\left(u_{l}\right) \geq\left(f_{u}^{0}\right)_{+}^{\prime}\left(u_{l}\right)
$$

$$
H_{l-}^{\delta}(x, t)=\left\{\begin{array}{lll}
\delta_{1}, & -\infty & <x / t<\frac{f\left(y_{1}\right)-f\left(u_{l}\right)}{y_{1}-u_{l}} \\
u_{l}-y_{1}, & \frac{f\left(y_{1}\right)-f\left(u_{l}\right)}{\left.y_{1}-u_{l}\right)} & <x / t<\frac{f\left(y_{1}\right)-f\left(u_{l}+\delta_{1}\right)}{y_{1}-u_{l}-\delta_{1}} \\
0, & \frac{\left.f y_{1}\right)-f\left(u_{l}+\delta_{1}\right)}{y_{1}-u_{l}-\delta_{1}} & <x / t<\infty .
\end{array}\right.
$$

If (32) is violated, we have

$$
\begin{aligned}
& H_{l-}^{\delta}(x, t)= \begin{cases}\delta_{1}, \quad-\infty & <x / t<\frac{f\left(u_{l}\right)-f\left(u_{l}+\delta_{1}\right)}{-\delta_{1}} \\
0, \quad \frac{f\left(u_{l}\right)-f\left(u_{l}+\delta_{1}\right)}{-\delta_{1}} & <x / t<\frac{f\left(y^{1}\right)-f\left(u^{l}\right)}{y^{1}-u^{l}} \\
0, \quad \frac{f\left(y^{1}\right)-f\left(u^{l}\right)}{y^{1}-u^{l}} & <x / t<\infty\end{cases} \\
& \text { If }\left\{f_{+}^{\prime}\left(u_{l}\right)=\left(f_{u}^{0}\right)_{+}^{\prime}\left(u_{l}\right) \text { or for all } u \in\left(u_{l}, y_{1}\right) \text { we have } f(u)>f_{u}^{0}(u)\right\}
\end{aligned}
$$

then

If (33) is violated, we have

$$
H_{l+}^{\delta}(x, t)=\left\{\begin{array}{lll}
\delta_{1}, & -\infty & <x / t<\frac{f\left(y_{1}\right)-f\left(u_{l}+\delta_{1}\right)}{y_{1}-u_{l}-\delta_{1}} \\
y_{1}-u_{l}, & \frac{f\left(y_{1}\right)-f\left(u_{l}+\delta_{1}\right)}{y_{1}-u_{l}-\delta_{1}}<x / t<\frac{f\left(y_{1}\right)-f\left(u_{l}\right)}{y_{1}-u_{l}} \\
0, & \frac{f\left(y_{1}\right)-f\left(u_{l}\right)}{y_{1}-u_{l}} & <x / t<\infty .
\end{array}\right.
$$

$$
H_{l+}^{\delta}(x, t)=\left\{\begin{array}{lll}
\delta_{1}, & -\infty & <x / t<\frac{f(\bar{u})-f\left(u_{l}+\delta_{1}\right)}{u-u_{l}-\delta_{1}} \\
\bar{u}-u_{l}, & \frac{f(\bar{u})-f\left(u_{l}+\delta_{1}\right)}{\left.\bar{u}-u_{l}-\delta_{1}\right)}<x / t<\frac{f\left(y_{1}\right)-f\left(u_{l}\right)}{y_{1}-u_{l}} \\
0, & \frac{f\left(y_{1}\right)-f\left(u_{l}\right)}{y_{1}-u_{l}}<x / t<\infty .
\end{array}\right.
$$

we have

$$
\text { If } f_{+}^{\prime}\left(u_{r}\right) \leq\left(f_{u}^{0}\right)_{-}^{\prime}\left(u_{r}\right)
$$

$$
H_{r+}^{\delta}(x, t)=\left\{\begin{array}{lll}
0, & -\infty & <x / t<\frac{f\left(u_{r}+\delta_{2}\right)-f\left(y_{k}\right)}{u_{r}+\delta_{2}-y_{k}} \\
u_{r}-y_{k}, & \frac{f\left(u_{r}+\delta_{2}\right)-f\left(y_{k}\right)}{u_{r}+\delta_{2}-y_{k}}<x / t<\frac{f\left(u_{r}\right)-f\left(y_{k}\right)}{u_{r}-y_{k}} \\
\delta_{2}, & \frac{f\left(u_{r}\right)-f\left(y_{k}\right)}{u_{r}-y_{k}}<x / t<\infty .
\end{array}\right.
$$

If (34) is violated, we have

$$
H_{r+}^{\delta}(x, t)= \begin{cases}0, \quad-\infty & <x / t<\frac{f\left(u_{r}\right)-f\left(y_{k}\right)}{u_{r}-y_{k}} \\ 0, \quad \frac{f\left(u_{r}\right)-f\left(y_{k}\right)}{u_{r}-y_{k}} & <x / t<\frac{f\left(u_{r}+\delta_{2}\right)-f\left(u_{r}\right)}{\delta_{2}} \\ \delta_{2}, \quad \frac{f\left(u_{r}+\delta_{2}\right)-f\left(u_{r}\right)}{\delta_{2}}<x / t<\infty .\end{cases}
$$


we have

$$
\text { If }\left\{f_{-}^{\prime}\left(u_{r}\right)=\left(f_{u}^{0}\right)_{-}^{\prime}\left(u_{r}\right) \text { or for all } u \in\left(y_{k}, u_{r}\right) \text { we have } f(u)>f_{u}^{0}(u)\right\}
$$

If (35) is violated, we have

$$
H_{r-}^{\delta}(x, t)=\left\{\begin{array}{lll}
0, & -\infty & <x / t<\frac{f\left(u_{r}\right)-f\left(y_{k}\right)}{u_{r}-y_{k}} \\
y_{k}-u_{r}, & \frac{f\left(u_{r}\right)-f\left(y_{k}\right)}{u_{r}-y_{k}} & <x / t<\frac{f\left(u_{r}+\delta_{2}\right)-f\left(y_{k}\right)}{u_{r}+\delta_{2}-y_{k}} \\
\delta_{2}, & \frac{f\left(u_{r}+\delta_{2}\right)-f\left(y_{k}\right)}{u_{r}+\delta_{2}-y_{k}}<x / t<\infty .
\end{array}\right.
$$

$$
H_{r-}^{\delta}(x, t)=\left\{\begin{array}{lll}
0, & -\infty & <x / t<\frac{f\left(u_{r}\right)-f\left(y_{k}\right)}{u_{r}-y_{k}} \\
\bar{u}-u_{r}, & \frac{f\left(u_{r}\right)-f\left(y_{k}\right)}{u_{r}-y_{k}} & <x / t<\frac{f\left(u_{r}+\delta_{2}\right)-f(\bar{u})}{u_{r}+\delta_{2}-\bar{u}} \\
\delta_{2}, & \frac{f\left(u_{r}+\delta_{2}\right)-f(\bar{u})}{u_{r}+\delta_{2}-\bar{u}}<x / t<\infty .
\end{array}\right.
$$

Proof. First we compute $y^{\delta}$. The change from $u_{l}$ to $u_{l}+\delta_{1}$ causes a shift of the first vertex in the polygonal line that is the boundary of the convex hull of the set

$$
\left\{(y, v): u_{l}+\delta_{1} \leq y \leq u_{r}+\delta_{2}, v \geq f(y)\right\} .
$$

Moreover, if $\left|\delta_{1}\right|$ is sufficiently small, at most one additional vertex compared with the set (5) appears between the first and the second vertex.

The change from $u_{r}$ to $u_{r}+\delta_{2}$ causes a shift of the last vertex. Moreover, if $\left|\delta_{2}\right|$ is sufficiently small, at most one additional vertex compared with the set (5) appears between the last and the second to last vertex.

7.0.1. Case 1: Assume that $\delta_{1}<0$

Subcase 1a. Lemma 5.2 implies that if $\left|\delta_{1}\right|+\left|\delta_{2}\right|$ is sufficiently small, and (32) holds, the first vertex in the convex hull of the set (5) is shifted to $\left(u_{l}+\delta_{1}, f\left(u_{l}+\delta_{1}\right)\right)$ and no additional vertices are generated.

In this case we have

$$
y^{\delta}(x, t)=\left\{\begin{array}{lll}
u_{l}+\delta_{1}, & -\infty & <x / t<\frac{f\left(y^{1}\right)-f\left(u_{l}+\delta_{1}\right)}{y^{1}-u_{l}-\delta_{1}} \\
y^{1}, & \frac{f\left(y^{1}\right)-f\left(u_{l}+\delta_{1}\right)}{y^{1}-u_{l}-\delta_{1}} & <x / t<\frac{f\left(y^{2}\right)-f\left(y^{1}\right)}{y^{2}-y^{1}} \\
\vdots & &
\end{array}\right.
$$

Subcase 1b. Lemma 5.2 implies that if $\left|\delta_{1}\right|+\left|\delta_{2}\right|$ is sufficiently small, and (32) is violated, the first vertex in the convex hull of the set (5) is shifted to $\left(u_{l}+\delta_{1}, f\left(u_{l}+\delta_{1}\right)\right)$ and the point $\left(u_{l}, f\left(u_{l}\right)\right)$ appears as an additional vertex.

In this case we have

$$
y^{\delta}(x, t)=\left\{\begin{array}{lll}
u_{l}+\delta_{1}, & -\infty & <x / t<\frac{f\left(u_{l}\right)-f\left(u_{1}+\delta_{1}\right)}{-\delta_{1}} \\
u^{l}, & \frac{f\left(u_{l}\right)-f\left(u_{l}+\delta_{1}\right)}{-\delta_{1}}<x / t<\frac{f\left(y^{1}\right)-f\left(u^{l}\right)}{y^{1}-u^{l}} \\
y^{1}, & \frac{f\left(y^{1}\right)-f\left(u^{l}\right)}{y^{1}-u^{l}} & <x / t<\frac{f\left(y^{2}\right)-f\left(y^{1}\right)}{y^{2}-y^{1}} \\
\vdots & &
\end{array}\right.
$$

Detection of Subcase 1a/Subcase 1b. How can we detect the Subcase 1a/Subcase 1b respectively numerically?

Let $p$ be the largest breakpoint of $f$ that is less than $u_{l}$ if such a breakpoint exists, and $p=u_{1}-1$ otherwise.

If

$$
f_{-}^{\prime}\left(u_{l}\right)=\frac{f\left(u_{l}\right)-f(p)}{u_{l}-p} \geq \frac{f\left(y_{1}\right)-f\left(u_{l}\right)}{y_{1}-u_{l}}
$$

we are in Subcase 1a, otherwise we are in Subcase 1b. Note that the last differential quotient in (39) gives the right-hand side derivative in the point $u_{l}$ of the function that is defined by the boundary of the convex hull of the epigraph of $f$. 


\subsubsection{Case 2: Assume that $\delta_{1}>0$}

Subcase 2a. Lemma 5.2 implies that if $\left|\delta_{1}\right|+\left|\delta_{2}\right|$ is sufficiently small, and (33) holds, the first vertex in the convex hull of the set (5) is shifted to $\left(u_{l}+\delta_{1}, f\left(u_{l}+\delta_{1}\right)\right)$ and no additional vertices are generated.

In this case we have

$$
y^{\delta}(x, t)=\left\{\begin{array}{lll}
u_{l}+\delta_{1}, & -\infty & <x / t<\frac{f\left(y^{1}\right)-f\left(u_{l}+\delta_{1}\right)}{y^{1}-u_{l}-\delta_{1}} \\
y^{1}, & \frac{f\left(y^{1}\right)-f\left(u_{l}+\delta_{1}\right)}{y^{1}-u_{l}-\delta_{1}} & <x / t<\frac{f\left(y^{2}\right)-f\left(y^{1}\right)}{y^{2}-y^{1}} \\
\vdots & &
\end{array}\right.
$$

Note that $y^{\delta}(x, t)$ has the same form as in Subcase 1a.

Subcase 2b. Lemma 5.2 implies that if $\left|\delta_{1}\right|+\left|\delta_{2}\right|$ is sufficiently small, and (33) is violated, the first vertex in the convex hull of the set (5) is shifted to $\left(u_{l}+\delta_{1}, f\left(u_{l}+\delta_{1}\right)\right)$ and the point $(\bar{u}, f(\bar{u}))$ appears as an additional vertex.

In this case we have

$$
y^{\delta}(x, t)=\left\{\begin{array}{lll}
u_{l}+\delta_{1}, & -\infty & <x / t<\frac{f(\bar{u})-f\left(u_{l}+\delta_{1}\right)}{\bar{u}-u_{l}-\delta_{1}} \\
\bar{u}, & \frac{f(\bar{u})-f\left(u_{l}+\delta_{1}\right)}{\bar{u}-u_{l}-\delta_{1}}<x / t<\frac{f\left(y^{1}\right)-f(\bar{u})}{y^{1}-\bar{u}} \\
y^{1}, & \frac{f\left(y^{1}\right)-f(\bar{u})}{y^{1}-\bar{u}} & <x / t<\frac{f\left(y^{2}\right)-f\left(y^{l}\right)}{y^{2}-y^{l}} \\
\vdots & &
\end{array}\right.
$$

Detection of Subcase $\mathbf{2 a}$ /Subcase $\mathbf{2 b}$. In order to check wether we are in Subcase $2 \mathrm{~b}$, we have to verify wether the following condition holds:

$$
f_{+}^{\prime}\left(u_{l}\right)>\frac{f\left(y_{1}\right)-f\left(u_{l}\right)}{y_{1}-u_{l}}
$$

and there exists a breakpoint $\bar{u}$ of $f$ in the open interval $\left(u_{l}, y_{1}\right)$ such that

$$
\bar{u}=\bar{\lambda} u_{l}+(1-\bar{\lambda}) y_{1}, f(\bar{u})=\bar{\lambda} f\left(u_{l}\right)+(1-\bar{\lambda}) f\left(y_{1}\right) .
$$

If (42) and (43) hold, we are in Subcase 2b, otherwise we are in Subcase 2a.

In Subcase $2 \mathrm{~b}$ we have to determine the smallest $\bar{u}$ satisfying (43). This is in fact a finite optimization problem since we only have to compare the values of $f$ and the lower convex envelope of $f$ on $\left(u_{l}, y_{1}\right)$ at the breakpoints of $f$ in $\left(u_{l}, y_{1}\right)$.

Now we analyze analogously the changes that can occur as a result of the shift from $u_{r}$ to $u_{r}+\delta_{2}$ if $\left|\delta_{2}\right|$ is sufficiently small. Again we have overall four cases.

\subsubsection{Case 1: Assume that $\delta_{2}>0$}

Subcase 1a. Lemma 5.2 implies that if $\left|\delta_{1}\right|+\left|\delta_{2}\right|$ is sufficiently small, and (34) holds, the last vertex in the convex hull of the set $(5)$ is shifted to $\left(u_{r}+\delta_{2}, f\left(u_{r}+\delta_{2}\right)\right)$ and no additional vertices are generated.

In this case we have

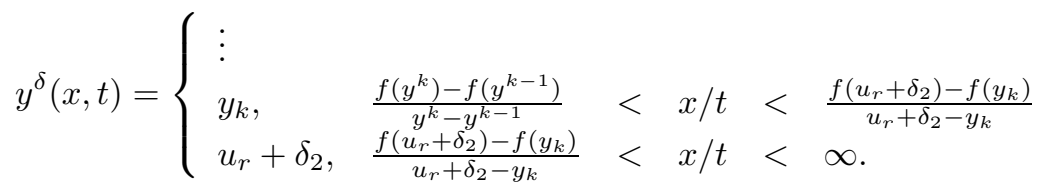

Subcase 1b. Lemma 5.2 implies that if $\left|\delta_{1}\right|+\left|\delta_{2}\right|$ is sufficiently small, and (34) is violated, the last vertex in the convex hull of the set (5) is shifted to $\left(u_{r}+\delta_{2}, f\left(u_{r}+\delta_{2}\right)\right)$ and the point $\left(u_{r}, f\left(u_{r}\right)\right)$ appears as an additional vertex. 
In this case we have

$$
y^{\delta}(x, t)=\left\{\begin{array}{lll}
\vdots & \\
y_{k}, & \frac{f\left(y^{k}\right)-f\left(y^{k-1}\right)}{y^{k}-y^{k-1}} & <x / t<\frac{f\left(u_{r}\right)-f\left(y_{k}\right)}{u_{r}-y_{k}} \\
u_{r}, & \frac{f\left(u_{r}\right)-f\left(y_{k}\right)}{u_{r}-y_{k}} & <x / t<\frac{f\left(u_{r}+\delta_{2}\right)-f\left(u_{r}\right)}{\delta_{2}} \\
u_{r}+\delta_{2}, & \frac{f\left(u_{r}+\delta_{2}\right)-f\left(u_{r}\right)}{\delta_{2}}<x / t<\infty .
\end{array}\right.
$$

Detection of Subcase 1a/Subcase 1b. If

$$
f_{+}^{\prime}\left(u_{r}\right)=\frac{f\left(u_{r}+\delta_{2}\right)-f\left(u_{r}\right)}{\delta_{2}} \leq \frac{f\left(u_{r}\right)-f\left(y_{k}\right)}{u_{r}-y_{k}}
$$

we are in Subcase 1a, otherwise we are in Subcase 1b. Note that the last differential quotient in (46) gives the left-hand side derivative in the point $u_{r}$ of the lower convex envelope of $f$ on $\left[u_{r}, u_{l}\right]$.

7.0.4. Case 2: Assume that $\delta_{2}<0$

Subcase 2a. Lemma 5.2 implies that if $\left|\delta_{1}\right|+\left|\delta_{2}\right|$ is sufficiently small, and (35) holds, the last vertex in the convex hull of the set $(5)$ is shifted to $\left(u_{r}+\delta_{2}, f\left(u_{r}+\delta_{2}\right)\right)$ and no additional vertices are generated.

In this case we have

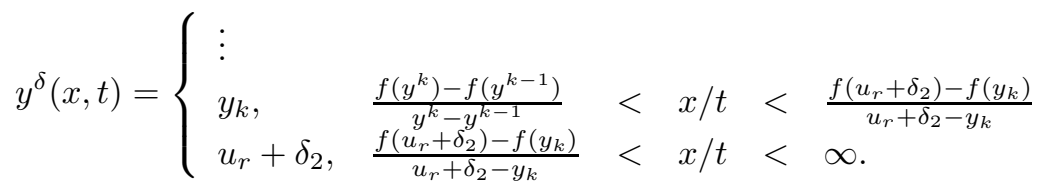

Note that $y^{\delta}(x, t)$ has the same form as in Subcase 1a.

Subcase 2b. Lemma 5.2 implies that if $\left|\delta_{1}\right|+\left|\delta_{2}\right|$ is sufficiently small, and (35) is violated, the last vertex in the convex hull of the set (5) is shifted to $\left(u_{r}+\delta_{2}, f\left(u_{r}+\delta_{2}\right)\right)$ and a point $(\bar{u}, f(\bar{u}))$ appears as an additional vertex. This point $\bar{u}$ is a breakpoint of $f$ that can be found as follows:

There exists $\bar{\lambda} \in(0,1)$ such that

$$
f\left(\bar{\lambda} y_{k}+(1-\bar{\lambda}) u_{r}\right)=\bar{\lambda} f\left(y_{k}\right)+(1-\bar{\lambda}) f\left(u_{r}\right) .
$$

Choose the largest $\bar{\lambda}$ of this type; in fact this $\bar{\lambda}$ is less than one. Define

$$
\bar{u}=\bar{\lambda} y_{k}+(1-\bar{\lambda}) u_{r}
$$

In this case we have

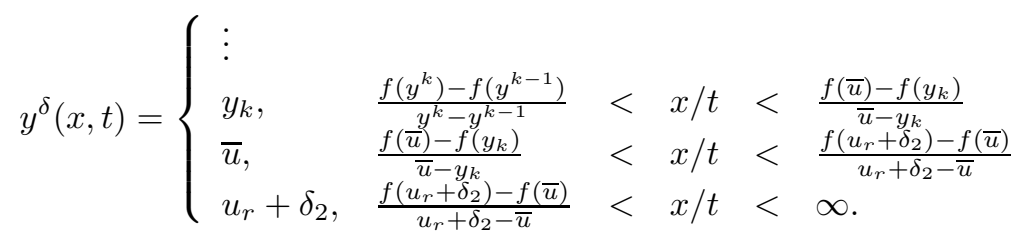

Detection of Subcase $2 \mathrm{a} /$ Subcase $2 \mathrm{~b}$. If

$$
f_{-}^{\prime}\left(u_{r}\right)<\frac{f\left(u_{r}\right)-f\left(y_{k}\right)}{u_{r}-y_{k}}
$$

and there exists a breakpoint $\bar{u}$ of $f$ in the open interval $\left(y_{k}, u_{r}\right)$ such that

$$
\bar{u}=\bar{\lambda} y_{k}+(1-\bar{\lambda}) u_{r}, \text { and } f(\bar{u})=\bar{\lambda} f\left(y_{k}\right)+(1-\bar{\lambda}) f\left(u_{r}\right),
$$


then we are in Subcase $2 \mathrm{~b}$.

If (48) or (49) is violated, we are in Subcase 2a.

\subsubsection{The expansion of $y^{\delta}$}

The above considerations show that in the expansion

$$
y^{\delta}=y+H^{\delta}
$$

we can decompose $H^{\delta}$ in the form

$$
H^{\delta}=H_{l}^{\delta}+H_{r}^{\delta},
$$

where $H_{l}^{\delta}$ gives the sensitivity with respect to $u_{l}$ and $H_{r}^{\delta}$ contains the sensitivity information for $u_{r}$. Moreover, we have to consider one-sided sensitivities $H_{l+}^{\delta}, H_{l-}^{\delta}, H_{r+}^{\delta}, H_{r-}^{\delta}$ corresponding to the cases $\delta_{1}>0, \delta_{1}<0, \delta_{2}>0, \delta_{2}<0$ respectively.

Define

$$
G^{\delta}(x, t)=y^{\delta}(x, t)-y(x, t)
$$

Variations of $\delta_{1}$. First we consider the effect of the variations of $\delta_{1}$. In Subcase 1a we have

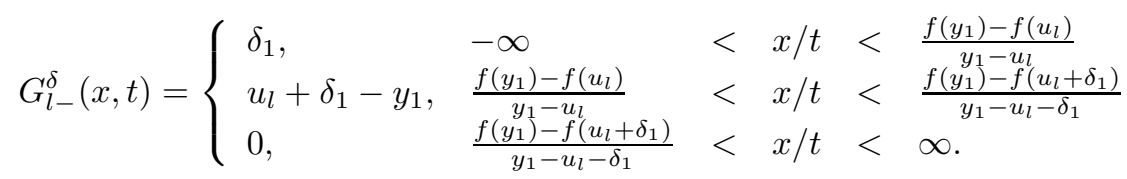

Note that

$$
\frac{f\left(y_{1}\right)-f\left(u_{l}\right)}{y_{1}-u_{l}} \leq \frac{f\left(y_{1}\right)-f\left(u_{l}+\delta_{1}\right)}{y_{1}-u_{l}-\delta_{1}}
$$

since $u_{l} \in\left[u_{l}+\delta_{1}, y_{1}\right]$ is not a breakpoint of the lower convex envelope of $f$ on $\left[u_{l}+\delta_{1}, y_{1}\right]$.

To obtain a first order approximation of $G_{l-}^{\delta}$ we define

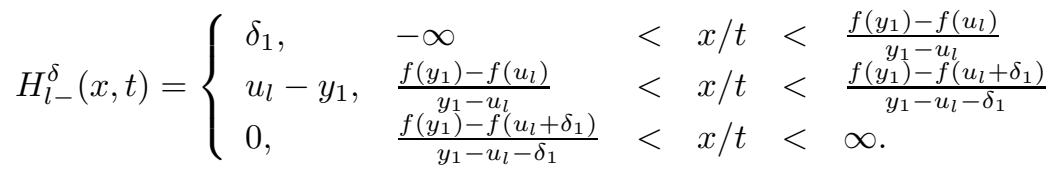

In Subcase $1 b$ we have

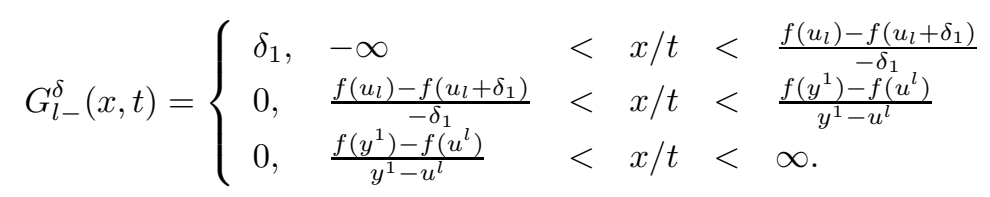

To obtain a first order approximation of $G_{l-}^{\delta}$ we define

$$
H_{l-}^{\delta}(x, t)=G_{l-}^{\delta}(x, t)
$$

In Subcase 2a we have

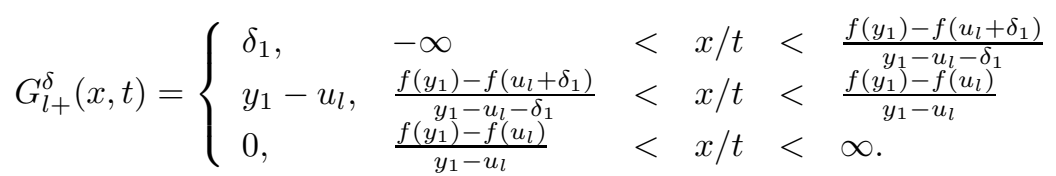

To obtain a first order approximation of $G_{l+}^{\delta}$ we define

$$
H_{l+}^{\delta}(x, t)=G_{l+}^{\delta}(x, t)
$$


In Subcase $2 b$ we have

$$
G_{l+}^{\delta}(x, t)=\left\{\begin{array}{lll}
\delta_{1}, & -\infty & <x / t<\frac{f(\bar{u})-f\left(u_{l}+\delta_{1}\right)}{\bar{u}-u_{l}-\delta_{1}} \\
\bar{u}-u_{l}, & \frac{f(\bar{u})-f\left(u_{l}+\delta_{1}\right)}{\bar{u}-u_{l}-\delta_{1}}<x / t<\frac{f\left(y_{1}\right)-f\left(u_{l}\right)}{y_{1}-u_{l}} \\
0, & \frac{f\left(y_{1}\right)-f\left(u_{l}\right)}{y_{1}-u_{l}} & <x / t<\infty .
\end{array}\right.
$$

To obtain a first order approximation of $G_{l+}^{\delta}$ we define

$$
H_{l+}^{\delta}(x, t)=G_{l+}^{\delta}(x, t)
$$

Variations of $\delta_{2}$. Now we consider the effect of the variations of $\delta_{2}$.

In Subcase 1a we have

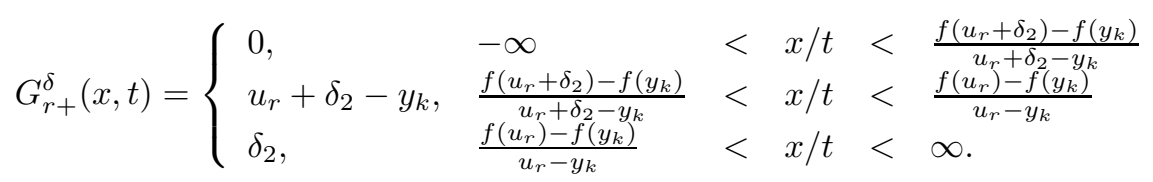

To obtain a first order approximation of $G_{r+}^{\delta}$, we define

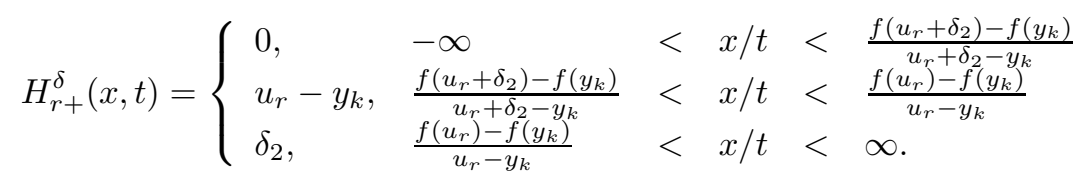

In Subcase 1b we have

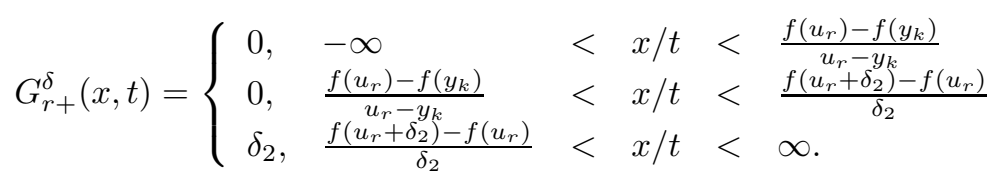

To obtain a first order approximation of $G_{r+}^{\delta}$, we define

$$
H_{r+}^{\delta}(x, t)=G_{r+}^{\delta}(x, t) .
$$

In Subcase 2a we have

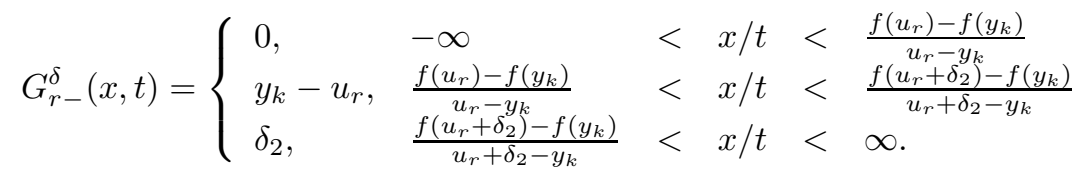

To obtain a first order approximation of $G_{r-}^{\delta}$, we define

$$
H_{r-}^{\delta}(x, t)=G_{r-}^{\delta}(x, t) .
$$

In Subcase $2 \mathrm{~b}$ we have

$$
G_{r-}^{\delta}(x, t)=\left\{\begin{array}{lll}
0, & -\infty & <x / t<\frac{f\left(u_{r}\right)-f\left(y_{k}\right)}{u_{r}-y_{k}} \\
\bar{u}-u_{r}, & \frac{f\left(u_{r}\right)-f\left(y_{k}\right)}{u_{r}-y_{k}} & <x / t<\frac{f\left(u_{r}+\delta_{2}\right)-f(\bar{u})}{u_{r}+\delta_{2}-\bar{u}} \\
\delta_{2}, & \frac{f\left(u_{r}+\delta_{2}\right)-f(\bar{u})}{u_{r}+\delta_{2}-\bar{u}}<x / t<\infty .
\end{array}\right.
$$

To obtain a first order approximation of $G_{r-}^{\delta}$, we define

$$
H_{r-}^{\delta}(x, t)=G_{r-}^{\delta}(x, t) .
$$




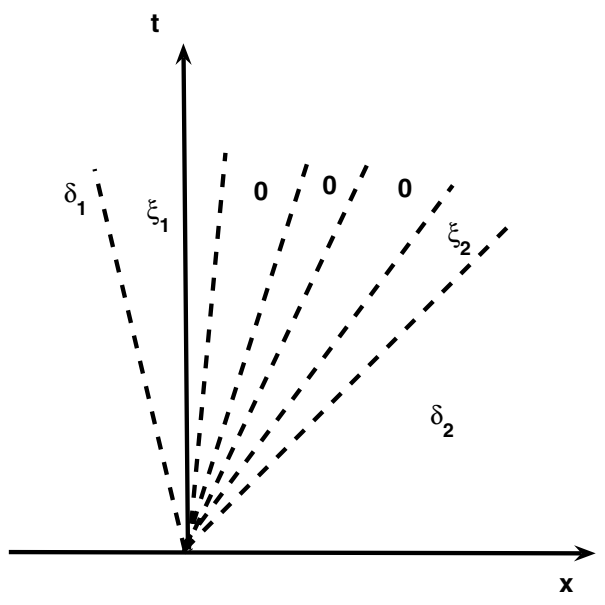

Figure 3. Structure of $H^{\delta}$ in $x-t$-plane. The states $\xi_{1}=0$ if (19) is satisfied and the value of the state $\xi_{2}$ depends on the condition (21).

7.0.6. Convergence of the expansion of $y^{\delta}$

Let

$$
\begin{aligned}
& l(\delta)=\max \left\{\frac{f\left(y_{1}\right)-f\left(u_{l}+\delta_{1}\right)}{y_{1}-u_{l}-\delta_{1}}, \frac{f\left(y_{1}\right)-f\left(u_{l}\right)}{y_{1}-u_{l}}\right\} \text { and } \\
& r(\delta)=\min \left\{\frac{f\left(u_{r}+\delta_{2}\right)-f\left(y_{k}\right)}{u_{r}+\delta_{2}-y_{k}}, \frac{f\left(u_{r}\right)-f\left(y_{k}\right)}{u_{r}-y_{k}}\right\} .
\end{aligned}
$$

Then we have

$$
\begin{gathered}
\left\|y^{\delta}-\left(y+H^{\delta}\right)\right\|_{L^{1}((0, T) \times \mathbb{R})}=\int_{0}^{T} \int_{\mathbb{R}}\left|y^{\delta}(x, t)-y(x, t)-H^{\delta}(x, t)\right| \mathrm{d} x \mathrm{~d} t \\
=\int_{0}^{T} \int_{-\infty}^{l(\delta) t}\left|G^{\delta}(x, t)-H^{\delta}(x, t)\right| \mathrm{d} x \mathrm{~d} t+\int_{0}^{T} \int_{r(\delta) t}^{\infty}\left|G^{\delta}(x, t)-H^{\delta}(x, t)\right| \mathrm{d} x \mathrm{~d} t \\
\leq \int_{0}^{T} \delta_{1} t\left|\frac{f\left(y_{1}\right)-f\left(u_{l}+\delta_{1}\right)}{y_{1}-u_{l}-\delta_{1}}-\frac{f\left(y_{1}\right)-f\left(u_{l}\right)}{y_{1}-u_{l}}\right| \mathrm{d} t \\
+\int_{0}^{T} \delta_{2} t\left|\frac{f\left(u_{r}+\delta_{2}\right)-f\left(y_{k}\right)}{u_{r}+\delta_{2}-y_{k}}-\frac{f\left(u_{r}\right)-f\left(y_{k}\right)}{u_{r}-y_{k}}\right| \mathrm{d} t \\
\leq \frac{T^{2}}{2}\left[\delta_{1}^{2} h_{1}\left(u_{l}, y_{1}, f\right)+\delta_{2}^{2} h_{2}\left(y_{k}, u_{r}, f\right)\right]
\end{gathered}
$$

with real numbers $h_{1}\left(u_{l}, y_{1}, f\right), h_{2}\left(y_{k}, u_{r}, f\right)$. Note that for $\delta_{1}$ only in Subcase 1a we have a contribution for $x / t \leq l(\delta)$ and for $\delta_{2}$ only in Subcase 1a we have a contribution for $x / t \geq r(\delta)$.

This finishes the proof.

Note that the shock structure of $H^{\delta}$ is similar to $y$ and $y^{\delta}$, respectively, except for at most two additional intervals. This allows an effective computation of $H^{\delta}$. In Figure 3 we give an example for the shock structure of $H^{\delta}$ in the $x-t$-plane. The value of the state $\xi_{1}$ depends whether the conditions (19) for $\delta_{1}<0$ and (20) for $\delta_{1}>0$ are satisfied or not. Similarly the state $\xi_{2}$ depends on the conditions (21) and (22) for $\delta_{2}>0$ and $\delta_{2}<0$, respectively.

By the above theorem we obtain that $H^{\delta}$ can be seen as surrogate for the first derivative of $y^{\delta}$ and hence can be used in optimization methods to compute the gradient. 


\section{Proof of Theorem 3.2}

In this section, we present the proof of Theorem 3.2.

Proof. If $D_{l}-f_{-}^{\prime}\left(u_{l}\right) \leq 0$, since $D_{l}=\left(f_{u}^{0}\right)_{+}^{\prime}\left(u_{l}\right)$, condition (32) holds. Hence we have for $\delta=\left(\delta_{1}, 0\right)$ with $\delta_{1}<0$

$$
\begin{aligned}
h(\delta)-h(0)= & \int_{-\infty}^{D_{l} T} g^{\prime}\left(u_{l}\right) \delta_{1} \chi_{\left[x_{1}, x_{2}\right]}(x) \mathrm{d} x \\
& +\int_{D_{l} T}^{\frac{f\left(y_{1}\right)-f\left(u_{l}+\delta_{1}\right)}{y_{1}-u_{l}-\delta_{1}} T}\left[g\left(u_{l}+\delta_{1}\right)-g\left(y_{1}\right)\right] \chi_{\left[x_{1}, x_{2}\right]}(x) \mathrm{d} x+O\left(\delta_{1}^{2}\right) \\
= & \int_{-\infty}^{D_{l} T} g^{\prime}\left(u_{l}\right) \delta_{1} \chi_{\left[x_{1}, x_{2}\right]}(x) \mathrm{d} x \\
& +\int_{D_{l} T}^{\frac{f\left(y_{1}\right)-f\left(u_{l}+\delta_{1}\right)}{y_{1}-u_{l}-\delta_{1}} T}\left[g\left(u_{l}\right)+g^{\prime}\left(u_{l}\right) \delta_{1}-g\left(y_{1}\right)\right] \chi_{\left[x_{1}, x_{2}\right]}(x) \mathrm{d} x+O\left(\delta_{1}^{2}\right) \\
= & g^{\prime}\left(u_{l}\right) \delta_{1} \mu\left(\left[x_{1}, x_{2}\right] \cap\left(-\infty, T D_{l}\right]\right) \\
& +\left[g\left(u_{l}\right)-g\left(y_{1}\right)+g^{\prime}\left(u_{l}\right) \delta_{1}\right] \mu\left(\left[x_{1}, x_{2}\right] \cap\left[D_{l} T, \frac{f\left(y_{1}\right)-f\left(u_{l}+\delta_{1}\right)}{y_{1}-u_{l}-\delta_{1}} T\right]\right)+O\left(\delta_{1}^{2}\right) .
\end{aligned}
$$

On account of (29) we have

$$
\begin{aligned}
& \mu\left(\left[x_{1}, x_{2}\right] \cap\left[D_{l} T, \frac{f\left(y_{1}\right)-f\left(u_{l}+\delta_{1}\right)}{y_{1}-u_{l}-\delta_{1}} T\right]\right) \\
& =\mu\left(\left[x_{1}, x_{2}\right] \cap\left[D_{l} T, D_{l} T+\delta_{1} \frac{D_{l}-f_{-}^{\prime}\left(u_{l}\right)}{y_{1}-u_{l}} T+O\left(\delta_{1}^{2}\right)\right]\right) \\
& =\delta_{1} \frac{D_{l}-f_{-}^{\prime}\left(u_{l}\right)}{y_{1}-u_{l}} T \chi_{\left[x_{1}, x_{2}\right)}\left(D_{l} T\right)+O\left(\delta_{1}^{2}\right)
\end{aligned}
$$

and this implies (11).

If $(32)$ is violated, we have for $\delta=\left(\delta_{1}, 0\right)$ with $\delta_{1}<0$

$$
\begin{aligned}
h(\delta)-h(0) & =\int_{-\infty}^{\frac{f\left(u_{l}\right)-f\left(u_{l}+\delta_{1}\right)}{-\delta_{1}} T} g^{\prime}\left(u_{l}\right) \delta_{1} \chi_{\left[x_{1}, x_{2}\right]}(x) \mathrm{d} x \\
& =g^{\prime}\left(u_{l}\right) \delta_{1} \mu\left(\left[x_{1}, x_{2}\right] \cap\left(-\infty, \frac{f\left(u_{l}\right)-f\left(u_{l}+\delta_{1}\right)}{-\delta_{1}} T\right]\right) \\
& =g^{\prime}\left(u_{l}\right) \delta_{1} \mu\left(\left[x_{1}, x_{2}\right] \cap\left(-\infty, f_{-}^{\prime}\left(u_{l}\right) T\right]\right)+O\left(\delta_{1}^{2}\right) .
\end{aligned}
$$

This implies (12).

If $\left\{D_{l}=f_{+}^{\prime}\left(u_{l}\right)\right.$ or for all $\left.u \in\left(u_{l}, y_{1}\right): f(u)>f_{u}^{0}(u)\right\}$, then (33) holds and we have for $\delta=\left(\delta_{1}, 0\right)$ with $\delta_{1}>0$

$$
\begin{aligned}
h(\delta)-h(0)= & \int_{-\infty}^{\frac{f\left(y_{1}\right)-f\left(u_{l}+\delta_{1}\right)}{y_{1}-u_{l}-\delta_{1}} T} g^{\prime}\left(u_{l}\right) \delta_{1} \chi_{\left[x_{1}, x_{2}\right]}(x) \mathrm{d} x \\
& +\int_{\frac{f\left(y_{1}\right)-f\left(u_{l}+\delta_{1}\right)}{y_{1}-u_{l}-\delta_{1}} T}^{D_{l} T}\left[g\left(y_{1}\right)-g\left(u_{l}\right)\right] \chi_{\left[x_{1}, x_{2}\right]}(x) \mathrm{d} x+O\left(\delta_{1}^{2}\right) \\
= & g^{\prime}\left(u_{l}\right) \delta_{1} \mu\left(\left[x_{1}, x_{2}\right] \cap\left(-\infty, T D_{l}\right]\right) \\
& +\left[g\left(y_{1}\right)-g\left(u_{l}\right)\right] \mu\left(\left[x_{1}, x_{2}\right] \cap\left[D_{l} T+\delta_{1} \frac{D_{l}-f_{+}^{\prime}\left(u_{l}\right)}{y_{1}-u_{l}} T, D_{l} T\right]\right)+O\left(\delta_{1}^{2}\right)
\end{aligned}
$$

where for the last step we have used (30). This implies (13). 
If (33) is violated, then

$$
\frac{f(\bar{u})-f\left(u_{l}\right)}{\bar{u}-u_{l}}=D_{l},
$$

and for $\delta=\left(\delta_{1}, 0\right)$ with $\delta_{1}>0$ we have

$$
\begin{aligned}
h(\delta)-h(0)= & \int_{-\infty}^{\frac{f(\bar{u})-f\left(u_{l}+\delta_{1}\right)}{\bar{u}-u_{l}-\delta_{1}} T} g^{\prime}\left(u_{l}\right) \delta_{1} \chi_{\left[x_{1}, x_{2}\right]}(x) \mathrm{d} x \\
& +\int_{\frac{f(\bar{u})-f\left(u_{l}+\delta_{1}\right)}{\bar{u}-u_{l}-\delta_{1}} T}^{D_{l} T}\left[g(\bar{u})-g\left(u_{l}\right)\right] \chi_{\left[x_{1}, x_{2}\right]}(x) \mathrm{d} x+O\left(\delta_{1}^{2}\right) \\
= & g^{\prime}\left(u_{l}\right) \delta_{1} \mu\left(\left[x_{1}, x_{2}\right] \cap\left(-\infty, T D_{l}\right]\right) \\
& +\left[g(\bar{u})-g\left(u_{l}\right)\right] \mu\left(\left[x_{1}, x_{2}\right] \cap\left[D_{l} T+\delta_{1} \frac{D_{l}-f_{+}^{\prime}\left(u_{l}\right)}{\bar{u}-u_{l}} T, D_{l} T\right]\right)+O\left(\delta_{1}^{2}\right) .
\end{aligned}
$$

where for the last step we have used (30). This implies (13).

If $f_{+}^{\prime}\left(u_{r}\right) \leq D_{r}$ since $D_{r}=\left(f_{u}^{0}\right)_{-}^{\prime}\left(u_{r}\right)$ condition (34) holds. Hence for $\delta=\left(0, \delta_{2}\right)$ with $\delta_{2}>0$ we have

$$
\begin{aligned}
h(\delta)-h(0)= & \int_{\frac{f\left(u_{r}+\delta_{2}\right)-f\left(y_{k}\right)}{u_{r}+\delta_{2}-y_{k}} T}^{D_{r} T}\left[g\left(u_{r}+\delta_{2}\right)-g\left(y_{k}\right)\right] \chi_{\left[x_{1}, x_{2}\right]}(x) \mathrm{d} x \\
& +\int_{D_{r} T}^{\infty} g^{\prime}\left(u_{r}\right) \delta_{2} \chi_{\left[x_{1}, x_{2}\right]}(x) \mathrm{d} x+O\left(\delta_{2}^{2}\right) \\
= & \int_{\frac{f\left(u_{r}+\delta_{2}\right)-f\left(y_{k}\right)}{u_{r}+\delta_{2}-y_{k}} T}^{D_{r} T}\left[g\left(u_{r}\right)+g^{\prime}\left(u_{r}\right) \delta_{2}-g\left(y_{k}\right)\right] \chi_{\left[x_{1}, x_{2}\right]}(x) \mathrm{d} x \\
& +\int_{D_{r} T}^{\infty} g^{\prime}\left(u_{r}\right) \delta_{2} \chi_{\left[x_{1}, x_{2}\right]}(x) \mathrm{d} x+O\left(\delta_{2}^{2}\right) \\
= & g^{\prime}\left(u_{r}\right) \delta_{2} \mu\left(\left[x_{1}, x_{2}\right] \cap\left(T D_{r}, \infty\right]\right) \\
& +\left[g\left(u_{r}\right)-g\left(y_{k}\right)+g^{\prime}\left(u_{r}\right) \delta_{2}\right] \mu\left(\left[x_{1}, x_{2}\right] \cap\left[\frac{f\left(y_{k}\right)-f\left(u_{r}+\delta_{2}\right)}{y_{k}-u_{r}-\delta_{2}} T, D_{r} T\right]\right)+O\left(\delta_{2}^{2}\right) .
\end{aligned}
$$

On account of (30) we have

$$
\begin{aligned}
& \mu\left(\left[x_{1}, x_{2}\right] \cap\left[\frac{f\left(y_{k}\right)-f\left(u_{r}+\delta_{2}\right)}{y_{k}-u_{r}-\delta_{2}} T, D_{r} T\right]\right) \\
& =\mu\left(\left[x_{1}, x_{2}\right] \cap\left[D_{r} T+\delta_{2} \frac{D_{r}-f_{+}^{\prime}\left(u_{r}\right)}{y_{k}-u_{r}} T+O\left(\delta_{2}^{2}\right), D_{r} T\right]\right) \\
& =-\delta_{2} \frac{D_{r}-f_{+}^{\prime}\left(u_{r}\right)}{y_{k}-u_{r}} T \chi_{\left(x_{1}, x_{2}\right]}\left(D_{r} T\right)+O\left(\delta_{2}^{2}\right)
\end{aligned}
$$

and this implies (17).

If $(34)$ is violated, we have for $\delta=\left(0, \delta_{2}\right)$ with $\delta_{2}>0$

$$
\begin{aligned}
h(\delta)-h(0) & =\int_{\frac{f\left(u_{r}+\delta_{2}\right)-f\left(u_{r}\right)}{\delta_{2}} T}^{\infty} g^{\prime}\left(u_{r}\right) \delta_{2} \chi_{\left[x_{1}, x_{2}\right]}(x) \mathrm{d} x+O\left(\delta_{2}^{2}\right) \\
& =g^{\prime}\left(u_{r}\right) \delta_{2} \mu\left(\left[x_{1}, x_{2}\right] \cap\left[\frac{f\left(u_{r}+\delta_{2}\right)-f\left(u_{r}\right)}{\delta_{2}} T, \infty\right)\right) \\
& =g^{\prime}\left(u_{r}\right) \delta_{2} \mu\left(\left[x_{1}, x_{2}\right] \cap\left[f_{+}^{\prime}\left(u_{r}\right) T, \infty\right)\right)+O\left(\delta_{2}^{2}\right) .
\end{aligned}
$$

This implies (18). 
If $\left\{D_{r}=f_{-}^{\prime}\left(u_{r}\right)\right.$ or for all $\left.u \in\left(y_{k}, u_{r}\right): f(u)>f_{u}^{0}(u)\right\}$, condition (35) holds and we have for $\delta=\left(0, \delta_{2}\right)$ with $\delta_{2}<0$

$$
\begin{aligned}
h(\delta)-h(0)= & \int_{D_{r} T}^{\frac{f\left(u_{r}+\delta_{2}\right)-f\left(y_{k}\right)}{u_{r}+\delta_{2}-y_{k}} T}\left[g\left(y_{k}\right)-g\left(u_{r}\right)\right] \chi_{\left[x_{1}, x_{2}\right]}(x) \mathrm{d} x \\
& +\int_{\frac{f\left(u_{r}+\delta_{2}\right)-f\left(y_{k}\right)}{u_{r}+\delta_{2}-y_{k}} T}^{\infty} g^{\prime}\left(u_{r}\right) \delta_{2} \chi_{\left[x_{1}, x_{2}\right]}(x) \mathrm{d} x+O\left(\delta_{2}^{2}\right) \\
= & g^{\prime}\left(u_{r}\right) \delta_{2} \mu\left(\left[x_{1}, x_{2}\right] \cap\left[\frac{f\left(u_{r}+\delta_{2}\right)-f\left(y_{k}\right)}{u_{r}+\delta_{2}-y_{k}} T, \infty\right)\right) \\
& +\left[g\left(y_{k}\right)-g\left(u_{r}\right)\right] \mu\left(\left[x_{1}, x_{2}\right] \cap\left[D_{r} T, \frac{f\left(u_{r}+\delta_{2}\right)-f\left(y_{k}\right)}{u_{r}+\delta_{2}-y_{k}} T\right]\right)+O\left(\delta_{2}^{2}\right) .
\end{aligned}
$$

On account of (29) we have

$$
\frac{f\left(u_{r}+\delta_{2}\right)-f\left(y_{k}\right)}{u_{r}+\delta_{2}-y_{k}}=D_{r}+\delta_{2} \frac{D_{r}-f_{-}^{\prime}\left(u_{r}\right)}{y_{k}-u_{r}}+O\left(\delta_{2}^{2}\right) .
$$

Thus

$$
h(\delta)-h(0)=g^{\prime}\left(u_{r}\right) \delta_{2} \mu\left(\left[x_{1}, x_{2}\right] \cap\left[D_{r} T, \infty\right)\right)+\left[g\left(y_{k}\right)-g\left(u_{r}\right)\right] \delta_{2} \chi_{\left[x_{1}, x_{2}\right)}\left(T D_{r}\right)\left(D_{r}-f_{-}^{\prime}\left(u_{r}\right)\right]+O\left(\delta_{2}^{2}\right) .
$$

This implies (15).

If (35) is violated, we have for $\delta=\left(0, \delta_{2}\right)$ with $\delta_{2}<0$

$$
\begin{aligned}
h(\delta)-h(0)= & \int_{D_{r} T}^{\frac{f\left(u_{r}+\delta_{2}\right)-f(\bar{u})}{u_{r}+\delta_{2}-\bar{u}} T}\left[g(\bar{u})-g\left(u_{r}\right)\right] \chi_{\left[x_{1}, x_{2}\right]}(x) \mathrm{d} x \\
& +\int_{\frac{f\left(u_{r}+\delta_{2}\right)-f(\bar{u})}{u_{r}+\delta_{2}-\bar{u}} T}^{\infty} g^{\prime}\left(u_{r}\right) \delta_{2} \chi_{\left[x_{1}, x_{2}\right]}(x) \mathrm{d} x+O\left(\delta_{2}^{2}\right) \\
= & g^{\prime}\left(u_{r}\right) \delta_{2} \mu\left(\left[x_{1}, x_{2}\right] \cap\left[\frac{f\left(u_{r}+\delta_{2}\right)-f(\bar{u})}{u_{r}+\delta_{2}-\bar{u}} T, \infty\right)\right) \\
& +\left[g\left(y_{k}\right)-g\left(u_{r}\right)\right] \mu\left(\left[x_{1}, x_{2}\right] \cap\left[D_{r} T, \frac{f\left(u_{r}+\delta_{2}\right)-f(\bar{u})}{u_{r}+\delta_{2}-\bar{u}} T\right]\right)+O\left(\delta_{2}^{2}\right) .
\end{aligned}
$$

On account of (29) and since $D_{r}=\left[f(\bar{u})-f\left(u_{r}\right)\right] /\left[\bar{u}-u_{r}\right]$ and we have

$$
\frac{f\left(u_{r}+\delta_{2}\right)-f(\bar{u})}{u_{r}+\delta_{2}-\bar{u}}=D_{r}+\delta_{2} \frac{D_{r}-f_{-}^{\prime}\left(u_{r}\right)}{y_{k}-u_{r}}+O\left(\delta_{2}^{2}\right) .
$$

Thus

$h(\delta)-h(0)=g^{\prime}\left(u_{r}\right) \delta_{2} \mu\left(\left[x_{1}, x_{2}\right] \cap\left[D_{r} T, \infty\right)\right)+\left[g(\bar{u})-g\left(u_{r}\right)\right] \delta_{2} \chi_{\left[x_{1}, x_{2}\right)}\left(T D_{r}\right)\left(D_{r}-f_{-}^{\prime}\left(u_{r}\right)\right]+O\left(\delta_{2}^{2}\right)$.

This implies (16).

\section{EXAMPLES}

The example presented are academic and should only illustrate the possible application of the derived calculus. The main advantage of the calculus is the application as a numerical method and as already mentioned we will present numerical results in a forthcoming publication. 


\subsection{Application to traffic flow problems}

We consider an (simplified) example related to traffic flow. A typical flux-function $f(\rho)$ in traffic flow applications is concave with a single maximum [9]. We normalize the maximal density to $\rho_{\max }=1$ and use in the following a piecewise linear approximation on $[0,1]$ of

$$
f(\rho)=\rho(1-\rho) .
$$

It is well-known that for Riemann initial data such that $u_{l} \leq \operatorname{argmax} f(\cdot)=\frac{1}{2}$ and $u_{r} \geq 1-u_{l}$, the solution to $(2),(3)$ is a shock wave travelling with non-positive velocity. We show that this information is also obtained by considering an optimal control problem and by discussing the necessary and sufficient optimality conditions derived by Theorem 3.2.

We fix $u_{l}<\frac{1}{2}$ and denote by $y$ the solution to $(2),(3)$ and (1). We consider the objective function given by

$$
I\left(u_{0}\right)=\frac{1}{2} \int_{-1}^{0}(y(x, T)-a)^{2} \mathrm{~d} x
$$

for $T$ suitable large and a constant $a>0$. We can control $u_{r}$, i.e., a part of the initial data for $u_{0}$ for $x \geq 0$. To be in the setting of the Theorem 3.2 we assume $u_{r} \geq u_{l}$.

A necessary condition for $u_{r}$ to be a minimizer is that the left-hand sided derivative $\partial_{\delta_{2}-} h(0) \leq 0$ and the right-hand sided derivative $\partial_{\delta_{2}} h(0) \geq 0$.

Then, $\partial_{\delta_{2}} h(0) \geq 0$ implies $u_{r} \geq 1-u_{l}$. Indeed, we distinguish the cases $(A)$ and $(B)$.

$$
\text { (A) } f_{+}^{\prime}\left(u_{r}\right)=1-2 u_{r} \leq D_{r}=1-\left(u_{r}+y_{k}\right) .
$$

This implies $u_{r} \geq y_{k}$ and due to the concavity of $f$ we obtain $y_{k} \equiv u_{l}$. For $T$ sufficiently large we obtain by $(17)$ and $\partial_{\delta_{2}} h(0) \geq 0, D_{r} \leq 0$ and $u_{r} \geq a$.

$$
\text { (B) } f_{+}^{\prime}\left(u_{r}\right)>D_{r}
$$

Then $u_{r}<y_{k}$ and by construction $u_{r}<y_{k} \cdots \leq u_{l}<\frac{1}{2}$, which contradicts the assumption $u_{r} \geq u_{l}$.

By $0 \geq \partial_{\delta_{2-}} h(0)$ we obtain similarly $0 \geq u_{r}-a$ by $(15)$ and $D_{r}<0$, which finally yields the expected, necessary optimality conditions for $u_{r}: u_{r}=a$ and $u_{r}>1-u_{l}$. This also shows, that the above is controllable, if and only if $a>1-u_{l}$ and $T$ sufficiently large.

Note that in this particularly simple situation, i.e., $u_{l}<\frac{1}{2}$ and $u_{r}>1-u_{l}$, the Front-Tracking solution consists of a single discontinuity traveling with the Rankine-Hugoniot speed $s=1-\left(u_{l}+\right.$ $\left.u_{r}\right)$. Hence, at each time $t>0$ we have the quantities $D_{r, l}, f_{ \pm}^{\prime}$ arising in main result (3.2) at hand without additional computational effort. This holds true not only in this particular case, but for all Front-Tracking approximations. In the setting of the previous example we have $f_{ \pm}^{\prime}\left(u_{r}\right)<D_{r}<0$ and $f(u)>f_{u}^{0}(u)$ for all $u \in\left(u_{l}, u_{r}\right)$. Therefore, the sensitivities with respect to $\delta_{2}$ are given by equations (17) and (15) and therefore we obtain up to order $O\left(\left\|\delta^{2}\right\|\right)$ and for $T$ sufficiently large

$$
I\left(u_{0}^{\delta}\right)=I\left(u_{0}\right)+\delta_{2} \partial_{\delta_{2 \pm}} h(0)=I\left(u_{0}\right)+\delta_{2}\left(u_{r}-a\right) .
$$

\subsection{Application to Burger's equation}

We consider Burger's equation

$$
\partial_{t} y+\partial_{x} \frac{1}{2} y^{2}=0
$$

with Riemann data $u_{0}(x)=\left(\begin{array}{ll}u_{l} & x<0 \\ u_{r} & x>0\end{array}\right)$. To apply the Front-Tracking algorithm (see Sect. 4) we approximate the flux function $\frac{1}{2} y^{2}$ by a continuous piecewise linear function $f(y)$ with breakpoints $\left\{y_{k}\right\}$. To keep the presentation simple, we assume that $u_{l}<0<u_{r}<1$ and $f(u)$ has the breakpoints $\left\{y_{1} \equiv u_{l}, y_{2} \equiv 0, y_{3} \equiv u_{r}\right\}$. Hence, the Front-Tracking solution to

$$
\partial_{t} y+\partial_{x} f(y)=0, y(x, 0)=u_{0}(x)
$$


consists of two discontinuities moving with the Rankine-Hugoniot speed $s_{1}=\frac{1}{2} u_{l}$ and $s_{2}=\frac{1}{2} u_{r}$, respectively.

Let us consider an objective functional of similar type as before with $T=1$ :

$$
I\left(u_{0}\right)=\frac{1}{2} \int_{0}^{1}(y(x, 1)-a)^{2} \mathrm{~d} x .
$$

Having the Front-Tracking solution with two discontinuities at hand, we immediately calculate $D_{r}=\frac{f\left(u_{r}\right)-f(0)}{u_{r}-0}=\frac{1}{2} u_{r}$ and $f_{-}^{\prime}\left(u_{r}\right)=D_{r}, f_{+}^{\prime}\left(u_{r}\right)=u_{r}>D_{r}$. Theorem 3.2 yields up to order $O\left(\|\delta\|^{2}\right)$ the sensitivities with respect to $\delta_{1}$ and $\delta_{2}$ as

$$
I\left(u_{0}^{\delta}\right)=I\left(u_{0}\right)+\delta_{2}\left(u_{r}-a\right)\left(1-u_{r}\right) .
$$

Note that $\partial_{\delta_{1 \pm}} h(0)=0$ since the wave-front $\left[u_{l}, 0\right]$ moves with negative velocity and therefore has no influence on the objective functional.

\section{Summary}

We derived a sensitivity calculus for the optimal control problem governed by nonlinear conservation laws with initial Riemann data. The calculus is valid also for non-convex, piecewise linear flux functions and general objective functionals. The generalization to piecewise constant control functions is straightforward and will be considered in a forthcoming paper containing also numerical results. The introduced calculus is easy to implement as numerical method for gradient computation due to the explicit formulas. Further it can be incorporated in existing Front-Tracking codes due to the similar structure of solutions.

Acknowledgements. This work has been partly supported by the Kaiserslautern Excellence Cluster Dependable Adaptive Systems and Mathematical Modeling and the DFG Project KL 1105/16-1 'Optimal Nodal Control Of Networked Systems Of Conservation Laws'.

\section{REFERENCES}

[1] A. Aw and M. Rascle, Resurrection of second order models of traffic flow? SIAM J. Appl. Math. 60 (2000) 916-938.

[2] A. Bressan, Hyperbolic Systems of Conservation Laws. Oxford University Press, Oxford (2000).

[3] C.M. Dafermos, Polygonal approximations of solutions of the initial value problem for a conservation law. $J$. Math. Anal. Appl. 38 (1972) 33-41.

[4] K. Ehrhardt and M. Steinbach, Nonlinear optimization in gas networks, in Modeling, Simulation and Optimization of Complex Processes, H.G. Bock, E. Kostina, H.X. Phu, R. Ranacher Eds. (2005) 139-148.

[5] M. Gugat, Optimal nodal control of networked hyperbolic systems: Evaluation of derivatives. AMO Advanced Modeling and Optimization 7 (2005) 9-37.

[6] M. Gugat, G. Leugering, K. Schittkowski and E.J.P.G. Schmidt, Modelling, stabilization and control of flow in networks of open channels, in Online optimization of large scale systems, M. Grötschel, S.O. Krumke, J. Rambau Eds., Springer (2001) 251-270.

[7] M. Gugat, G. Leugering and E.J.P.G. Schmidt, Global controllability between steady supercritical flows in channel networks. Math. Meth. Appl. Sci. (2003) 781-802.

[8] M. Gugat, M. Herty, A. Klar and G. Leugering, Optimal control for traffic flow networks. J. Optim. Theory Appl. 126 (2005) 589-616.

[9] D. Helbing, Verkehrsdynamik. Springer-Verlag, Berlin, Heidelberg, New York (1997).

[10] R. Holdahl, H. Holden and K.-A. Lie, Unconditionally stable splitting methods for the shallow water equations. BIT 39 (1999) 451-472.

[11] H. Holden and L. Holden, On scalar conservation laws in one-dimension, in Ideas and Methods in Mathematical Analysis, Stochastics and Applications S. Albeverio, J. Fenstad, H. Holden, T. Lindstrøm Eds. (1992) 480-509.

[12] H. Holden and N.H. Risebro, A mathematical model of traffic flow on a network of unidirectional roads. SIAM J. Math. Anal. 26 (1995) 999-1017.

[13] H. Holden and N.H. Risebro, Front tracking for hyperbolic conservation laws. Springer, New York, Berlin, Heidelberg (2002).

[14] H. Holden, L. Holden and R. Hoegh-Krohn, A numerical method for first order nonlinear scalar conservation laws in one-dimension. Comput. Math. Anal. 15 (1988) 595-602. 
[15] S.N. Kruzkov, First order quasi linear equations in several independent variables. Math. USSR Sbornik, 10 (1970) 217-243.

[16] R.J. LeVeque, Numerical methods for conservation laws. Birkhäuser Verlag, Basel, Boston, Berlin (1990).

[17] M.J. Lighthill and J.B. Whitham, On kinematic waves. Proc. Roy. Soc. Lond. A229 (1955) 281-345.

[18] J. Smoller, Shock waves and reaction diffusion equations. Springer, New York, Berlin, Heidelberg (1994).

[19] S. Ulbrich, A sensitivity and adjoint calculus for discontinuous solutions of hyperbolic conservation laws with source terms. SIAM J. Control Optim. 41 (2002) 740-797.

[20] S. Ulbrich, Adjoint-based derivative computations for the optimal control of discontinuous solutions of hyperbolic conservation laws. Syst. Control Lett. 3 (2003) 309-324.

To access this journal online: www.edpsciences.org 